NEW ZEALAND JOURNAL OF MATHEMATICS

Volume 52 (2021), 691-726

https://doi.org/10.53733/96

\title{
GLOBAL ASPECTS OF MEASURE PRESERVING EQUIVALENCE RELATIONS AND GRAPHS
}

\author{
Alexander S. KeChris
}

(Received 19 March 2021)

\begin{abstract}
This paper is an introduction and survey of a "global" theory of measure preserving equivalence relations and graphs. In this theory one views a measure preserving equivalence relation or graph as a point in an appropriate topological space and then studies the properties of this space from a topological, descriptive set theoretic and dynamical point of view.
\end{abstract}

Dedicated to the memory of Vaughan F.R. Jones

I had the chance to meet with Vaughan Jones on several occasions and I vividly recall some fascinating conversations, about mathematics and other subjects, that I had with him over the years. Vaughan was also instrumental in the creation of the New Zealand Mathematics Research Institute (NZMRI) and the organization of its summer meetings, since 1994, which brought together mathematicians from around the world to lecture and interact with New Zealand colleagues. I was very fortunate to participate in a wonderful such meeting in Algorithmic Information Theory, Computability and Complexity during January 2009 in Napier.

Although Vaughan Jones and I worked in different fields of mathematics, we shared a common interest in some aspects of ergodic theory, so it seemed appropriate to choose as the topic of this paper the study of measure preserving equivalence relations, an area in which Vaughan Jones, together with Klaus Schmidt, made a very influential contribution in $[\mathbf{J S}]$.

\section{Introduction}

A Polish space is a separable, completely metrizable topological space and a standard Borel space is a Polish space equipped with the $\sigma$-algebra of its Borel sets. Finally, a standard probability space is a measure space $(X, \mu)$, where $X$ is a standard Borel space and $\mu$ is a non-atomic, Borel probability measure on $X$. All standard probability spaces are measure-theoretically isomorphic to $([0,1], \lambda)$, where $\lambda$ is Lebesgue measure.

Suppose now that $\Gamma$ is a countable (discrete) group and $a: \Gamma \times X \rightarrow X$ a Borel action of $\Gamma$ on a standard Borel space $X$. Put $\gamma^{a}(x)=a(\gamma, x)$ and also $\gamma \cdot x=a(\gamma, x)$, when $a$ is understood. Moreover, we often use the notation $\Gamma \curvearrowright^{a} X$ or just $\Gamma \curvearrowright X$ for the action. Denote by $E_{a}$ the equivalence relation on $X$ generated by $a$ :

$$
x E_{a} y \Longleftrightarrow \exists \gamma \in \Gamma(\gamma \cdot x=y),
$$

whose classes are the orbits of the action. Sometimes when the action is understood, we also write $E_{\Gamma}^{X}$ instead of $E_{a}$. Clearly $E_{a}$ is a countable Borel equivalence relation (CBER), i.e., $E_{a} \subseteq X^{2}$ is Borel and every equivalence class is countable. Conversely, the Feldman-Moore Theorem asserts that if $E$ is a CBER on the standard Borel space $X$, then there is a countable group $\Gamma$ and a Borel action $a$ of 
$\Gamma$ on $X$ such that $E=E_{a}$. In fact there is a sequence of Borel involutions $\left(T_{n}\right)$ of $X$ that generates $E$, in the sense that $x E y \Longleftrightarrow \exists n\left(T_{n}(x)=y\right)$.

If now $(X, \mu)$ is a standard probability space and $a$ is a Borel action of $\Gamma$ on $X$, we say that $a$ is measure preserving if for every $\gamma \in \Gamma$ and every Borel set $A \subseteq X, \mu(\gamma \cdot A)=\mu(A)$. Two measure preserving actions $\Gamma \curvearrowright^{a} X$ and $\Delta \curvearrowright^{a} Y$, on standard probability spaces $(X, \mu),(Y, \nu)$, are orbit equivalent if there are invariant (under the corresponding actions) Borel sets $A \subseteq X, B \subseteq Y$ with $\mu(A)=\nu(B)=1$ and a Borel isomorphism $f: A \rightarrow B$ that sends $\mu$ to $\nu$ and satisfies $x E_{a} y \Longleftrightarrow f(x) E_{b} f(y)$. Neglecting null sets, as in common in measure theory, this simply says that $E_{a}, E_{b}$ are measure-theoretically isomorphic.This leads to the study of measure preserving equivalence relations on $(X, \mu)$. A CBER $E$ on $X$ is called measure preserving if it is of the form $E_{a}$ for some measure preserving $\Gamma \curvearrowright^{a} X$. It is easy to see that this notion is independent of the group and the action that generates $E$. In this context, we also say that $\mu$ is $E$-invariant. Again we identify measure preserving $E, F$ if they are measure-theoretically equal, i.e., if there is a Borel set $A \subseteq X$ with $\mu(A)=1$ such that $E|A=F| A$, where for any equivalence relation $R$ on a set $Z$ and any $C \subseteq Z$, we let $R \mid C=R \cap C^{2}$ be the restriction of $R$ to $C$. Note that we can assume that such a set $A$ is invariant under both $E, F$, i.e., $x \in A, x E y \Longrightarrow y \in A$ and similarly for $F$.

The study of orbit equivalence of measure preserving actions and also measure preserving equivalence relations is a very active area of research in ergodic theory and operator algebras, see for example $[\mathbf{Z}],[\mathbf{A P}]$, and $[\mathbf{I}]$, which also contains an extensive bibliography of work in this area over the last few decades.

We will also consider locally countable, Borel (simple, undirected) graphs on standard Borel spaces $X$. These are Borel subsets of $X^{2}$ that are symmetric, avoid the diagonal and have countable sections. For any such graph $G$ denote by $G^{*}$ the equivalence relation generated by $G$, whose classes are the connected components of $G$. Thus $G^{*}$ is a CBER and we say that $G$ is a graphing of $G^{*}$. When $(X, \mu)$ is a standard probability space, we say that $G$ is measure preserving if $G^{*}$ is measure preserving. Again we identify two such graphs $G, H$ if they agree $\mu$-a.e., i.e., there is a Borel $G^{*}$ - and $H^{*}$-invariant set $A$ with $\mu(A)=1$, such that $G|A=H| A$, where for any graph $F$ on a set $Z$ and any $C \subseteq Z$, we let $F \mid C=F \cap C^{2}$ be the induced subgraph on $C$. Ergodic theory also studies such measure preserving graphs.

Over the last few years I have been interested in developing a "global" theory of measure preserving equivalence relations and graphs. The idea is to consider a measure preserving equivalence relation or graph as a point in an appropriate topological space and then study the properties of this space from a topological, descriptive set theoretic and dynamical point of view. This work in progress is contained in the preprint $[\mathbf{K 3}]$ and the goal of the present paper is to present a survey of the current state of affairs in this subject and its open problems, referring to [K3] for a more complete development, including detailed proofs of all the results discussed here.

Acknowledgements. Preparation of this paper was supported by NSF Grant DMS-1950475. I would like to thank Lewis Bowen for asking some questions that got me thinking about this subject. Thanks also to Lewis, Peter Burton, Clinton Conley, François Le Maître, Ben Miller, Forte Shinko, Anush Tserunyan and Robin 
Tucker-Drob for many useful comments and corrections or for allowing me to include some results of theirs in $[\mathbf{K} 3]$ and quoted in this paper.

\section{Preliminaries}

Consider a standard probability space $(X, \mu)$. As usual we will neglect null sets in the sequel if there is no danger of confusion. We denote by $\operatorname{Aut}(X, \mu)$ the group of all Borel automorphisms of $X$ which preserve the measure $\mu$ and in which we identify two such automorphisms if they agree $\mu$-a.e. The weak topology on $\operatorname{Aut}(X, \mu)$ is the topology generated by the functions

$$
\operatorname{Aut}(X, \mu) \ni T \mapsto T(A), A \subseteq X \text { Borel }
$$

(i.e., the smallest topology making these functions continuous). We denote this topology by $w$. The group $\operatorname{Aut}(X, \mu)$ with this topology is a Polish topological group.

The group $\operatorname{Aut}(X, \mu)$ also admits the 2 -sided invariant (but not separable) metric:

$$
d_{u}(S, T)=\mu(\{x \in X: T(x) \neq S(x)\}),
$$

called the uniform metric. The associated topology on $\operatorname{Aut}(X, \mu)$ is called the uniform topology, denoted by $u$. We have $w \varsubsetneqq u$.

For a measure preserving CBER $E$ on $(X, \mu)$, its full group $[E]$ is the subgroup of all $T \in \operatorname{Aut}(X, \mu)$ such that $T(x) E x, \mu$-a.e. It is closed in the uniform topology and the metric $d_{u}$ restricted to $[E]$ is separable, so in the uniform topology $[E]$ is a Polish group admitting a 2-sided invariant metric.

Denote also by $[[E]]$ the full pseudogroup of $E$, i.e., the set of all partial Borel bijections $\varphi: A \rightarrow B$ with $\varphi(x) E x, \forall x \in A$. As usual we identify two such partial bijections if they agree $\mu$-a.e.

It is an interesting question to characterize the groups $[E]$ among the subgroups of the topological group $(\operatorname{Aut}(X, \mu), u)$. It can be shown that for any non-trivial involution $T \in \operatorname{Aut}(X, \mu)$, the centralizer of $T$ in $\operatorname{Aut}(X, \mu)$ has a largest abelian normal subgroup denoted by $A_{T}$. We now have:

Theorem 1.1. The following are equivalent for a subgroup $G$ of $\operatorname{Aut}(X, \mu)$ :

(i) $G=[E]$, for a measure preserving CBER $E$,

(ii) (a) $G$ is closed and separable in $(\operatorname{Aut}(X, \mu), u)$, (b) the group generated by the involutions in $G$ is uniformly dense in $G$, and (c) for every nontrivial involution $T \in G, A_{T} \subseteq G$.

Note that this characterization depends on the properties of $G$ within $(\operatorname{Aut}(X, \mu), u)$ and one can ask if there is a characterization depending only on the topological group structure of $(G, u)$. A strong negative answer is given in $\S 3$ of $[\mathbf{K 3}]$.

\section{The Topological Space of Subequivalence Relations}

We will denote by $\mathcal{E}$ the set of all measure preserving CBER on the standard probability space $(X, \mu)$ (recall here that two measure preserving CBER are identified if they agree $\mu$-a.e.). We would like to equip $\mathcal{E}$ with a nice topological structure but there is a difficulty here. As discussed in Section 4.1 there is no "definable" separable, metrizable topology on $\mathcal{E}$. On the other hand there is a way of defining 
a canonical topology on each "localization" of $\mathcal{E}$. More precisely, for each measure preserving CBER $E$ denote by $S(E)$ the set of subequivalence relations of $E$ :

$$
S(E)=\{F \in \mathcal{E}: F \subseteq E\},
$$

where for $E, F \in \mathcal{E}, F \subseteq E$ means that there is an invariant under $E$ Borel set $A$ with $\mu(A)=1$ such that for $x, y \in A$, we have $x F y \Longrightarrow x E y$, i.e., $F$ is contained in $E, \mu$-a.e. We will define a canonical Polish topology on each $S(E)$ and study this "localized" space. It will turn out that these topologies cohere under the inclusion $E \subseteq E^{\prime}$ and thus define a weak topology on $\mathcal{E}$ which is Hausdorff but not separable or first countable. Thus our study will concentrate on the structure of the "localized" spaces $S(E)$.

So from now on we will fix $E \in \mathcal{E}$ and define the canonical topology of $S(E)$. In fact we will give a number of equivalent descriptions of this topology.

2.1. The weak topology. Denote by $\mathrm{MALG}_{\mu}$ the measure algebra of $(X, \mu)$, i.e., the algebra of Borel sets in $X$ modulo null sets, which is a Polish Boolean algebra with the topology induced by the complete metric

$$
d_{\mu}(A, B)=\mu(A \triangle B),
$$

where $\triangle$ is symmetric difference. For each $T \in[E], F \in S(E)$, let

$$
A_{T, F}=\{x \in X:(x, T(x)) \in F\} \in \mathrm{MALG}_{\mu}
$$

Consider then the topology of $S(E)$ generated by the functions

$$
S(E) \ni F \mapsto \mu\left(A_{T, F}\right), T \in[E] .
$$

It turns out that this is a Polish topology on $S(E)$, which we call the weak topology.

2.2. The strong topology. Consider now the topology on $S(E)$ generated by the functions

$$
S(E) \ni F \mapsto A_{T, F} \in \mathrm{MALG}_{\mu}, T \in[E] .
$$

This is again a Polish topology on $S(E)$, which we call the strong topology.

Remark 2.1. For comparison, we note that $F_{n} \rightarrow F$ in the weak topology iff $\forall T \in[E]\left(\mu\left(A_{T, F_{n}}\right) \rightarrow \mu\left(A_{T, F}\right)\right)$, while $F_{n} \rightarrow F$ in the strong topology iff $\forall T \in$ $[E]\left(\mu\left(A_{T, F_{n}} \triangle A_{T, F}\right) \rightarrow 0\right)$.

2.3. A topology from the measure algebra of $E$. Below view $E$ as a genuine CBER on $X$ and not one viewed $\mu$-a.e. Then define a $\sigma$-finite Borel measure on $E \subseteq X^{2}$ as follows:

$$
M(W)=\int\left|W_{x}\right| d \mu(x)=\int\left|W^{y}\right| d \mu(y)
$$

for Borel $W \subseteq E$, where $W_{x}=\{y \in X:(x, y) \in W\}, W^{y}=\{x \in X:(x, y) \in W\}$ and $|A| \in \mathbb{N} \cup\{\infty\}$ is the cardinality of a set $A$. (The equality of the two integrals above follows from the assumption that $E$ is measure preserving.) There is a probability Borel measure $\nu$ on $E$ which is equivalent to $M$ (i.e., $M, \nu$ have the same null sets) and we define the measure algebra of $E$, in symbols MALG ${ }_{\mu, E}$, to be the measure algebra of $\nu$ with the associated topology. Both this algebra and the topology are independent of the choice of $\nu$ (although the metric that induces this topology depends on $\nu$ ) and it is also independent of the $\mu$-a.e. choice of $E$. 
Clearly $S(E)$ is a subspace of $\mathrm{MALG}_{\mu, E}$ and it turns out that it is actually closed, so we have the induced Polish topology on $S(E)$.

2.4. A topology related to invariant, random equivalence relations. Let $\Gamma$ be a countable (discrete) group and let $A(\Gamma, X, \mu)$ be the space of measure preserving actions of $\Gamma$ on $(X, \mu)$, where two actions are identified if they agree $\mu$-a.e.

We refer here to Section 12.1 for the definition of the space $\operatorname{Eq}(\Gamma)$ of equivalence relations on $\Gamma$, the space $\operatorname{IRE}(\Gamma)$ of invariant, random equivalence relations on $\Gamma$ and the maps $e_{F}^{a}: X \rightarrow \operatorname{Eq}(\Gamma)$, for $a \in A(\Gamma, X, \mu), F \in S\left(E_{a}\right)$. Fix $a \in A(\Gamma, X, \mu)$ with $E=E_{a}$. Consider the compact metrizable space $\mathcal{P}(\Gamma)^{\mathbb{N}} \times \operatorname{Eq}(\Gamma)$ (where $\mathcal{P}(\Gamma)$ is the space of all subsets of $\Gamma$, identified with $\left.2^{\Gamma}\right)$, on which $\Gamma$ acts continuously by $\gamma \cdot\left(\left(a_{n}\right), e\right)=\left(\left(\gamma a_{n}\right), \gamma \cdot e\right)$. Fix also a sequence $\left(D_{n}\right)$ of Borel sets which is dense in $\mathrm{MALG}_{\mu}$. Define then the $\Gamma$-equivariant map

$$
\theta_{F}^{a}=\theta_{F}: X \rightarrow \mathcal{P}(\Gamma)^{\mathbb{N}} \times \operatorname{Eq}(\Gamma),
$$

by $\theta_{F}(x)=\left(\left(a_{n}\right), e\right)$, where $a_{n}=\left\{\gamma: \gamma^{-1} \cdot x \in D_{n}\right\}$ and $e=e_{F}(x)$. Let $\tau^{a}(F)=$ $\tau(F)=\left(\theta_{F}\right)_{*} \mu \in \operatorname{Prob}\left(\mathcal{P}(\Gamma)^{\mathbb{N}} \times \operatorname{Eq}(\Gamma)\right)$, the space of Borel probability measures on $\left.\mathcal{P}(\Gamma)^{\mathbb{N}} \times \operatorname{Eq}(\Gamma)\right)$. Then $\tau(F)$ is $\Gamma$-invariant and so its projection on $\operatorname{Eq}(\Gamma)$ is in $\operatorname{IRE}(\Gamma)$.

Proposition 2.2. The map $\tau: S(E) \rightarrow \operatorname{Prob}\left(\mathcal{P}(\Gamma)^{\mathbb{N}} \times \operatorname{Eq}(\Gamma)\right)$ is a homeomorphism into $\operatorname{Prob}\left(\mathcal{P}(\Gamma)^{\mathbb{N}} \times \operatorname{Eq}(\Gamma)\right)$, where $S(E)$ is equipped with the weak topology.

Thus we can also view $S(E)$ as a $G_{\delta}$ subset of $\operatorname{Prob}\left(\mathcal{P}(\Gamma)^{\mathbb{N}} \times \operatorname{Eq}(\Gamma)\right)$ with its relative Polish topology.

2.5. A topology using measurable subgroups. (This is due to Robin TuckerDrob and the author. It is motivated by the idea of measurable subgroups, see [Bo, Section 4].)

First, without loss of generality, we can assume that $X=2^{\mathbb{N}}$ and $E$ is generated by a continuous action of a countable group $\Gamma$. (Recall here that we identify equivalence relations if they agree a.e.)

For $x \in X, F \in S(E)$, define $\Gamma_{x}^{F}=\left\{\gamma \in \Gamma:\left(x, \gamma^{-1} \cdot x\right) \in F\right\}$. Then $\Gamma_{x}^{F} \in$ $\mathcal{P}_{1}(\Gamma)=\{A \subseteq \Gamma: 1 \in A\}$. For $\gamma \in \Gamma, A \in \mathcal{P}_{1}(\Gamma)$, let $\gamma A=\{\gamma \delta: \delta \in A\}$. Put

$$
\varphi_{F}(x)=\left(x, \Gamma_{x}^{F}\right) \in X \times \mathcal{P}_{1}(\Gamma) .
$$

On $X \times \mathcal{P}_{1}(\Gamma)$ let

$$
(x, A) R(y, B) \Longleftrightarrow \exists \gamma \in \Gamma(\gamma \cdot x=y \& \gamma A=B) .
$$

Proposition 2.3. (i) $R$ is an equivalence relation.

(ii) $\varphi_{F}: X \rightarrow X \times \mathcal{P}_{1}(\Gamma)$ is $1-1$.

(iii) $\varphi_{F}(X)$ is $R$-invariant.

(iv) $x F y \Longleftrightarrow \varphi_{F}(x) R \varphi_{F}(y)$.

Since $\mu$ is $F$-invariant and $\varphi_{F}$ is a Borel bijection between $X$ and a Borel $R$ invariant subset of $X \times \mathcal{P}_{1}(\Gamma)$, it follows that $\left(\varphi_{F}\right)_{*} \mu=\mu_{F}$ is an $R$-invariant probability measure on $X \times \mathcal{P}_{1}(\Gamma)$.

Remark 2.4. Actually the definition of $\varphi_{F}, F \in S(E)$, depends on picking an a.e. representative for $F$ but it is easy to check that $\mu_{F}$ is well defined. 
Let $\mathcal{M}$ be the compact, metrizable space of probability measures on the compact zero-dimensional space $Y=X \times \mathcal{P}_{1}(\Gamma) \subseteq X \times \mathcal{P}(\Gamma)$. We also note the following.

Proposition 2.5. $\{\mu \in \mathcal{M}: \mu$ is $R$-invariant $\}$ is closed in $\mathcal{M}$.

Define now $\Phi: S(E) \rightarrow \mathcal{M}$ by $\Phi(F)=\mu_{F}=\left(\varphi_{F}\right)_{*} \mu$.

Proposition 2.6. The map $\Phi: S(E) \rightarrow \mathcal{M}$ is a homeomorphism of $S(E)$ into $\mathcal{M}$, where $S(E)$ is equipped with the weak topology.

Thus the space $S(E)$ can be identified with a $G_{\delta}$ subspace of $\mathcal{M}$ with its relative Polish topology.

2.6. A topology using actions of the free group $\mathbb{F}_{\infty}$. (This is due to Peter Burton) Denote by $(A(\Gamma, X, \mu), u)$ the space of measure preserving actions of $\Gamma$ on $(X, \mu)$ with the uniform topology $u$ (see $[\mathbf{K}$, Section $10,(\mathbf{A})]$ ). Here we consider the product topology on $\operatorname{Aut}(X, \mu)^{\Gamma}$, where $\operatorname{Aut}(X, \mu)$ is given the uniform topology. The space $A(\Gamma, X, \mu)$ is then viewed as a closed subspace of $\operatorname{Aut}(X, \mu)^{\Gamma}$ in this product topology. Given an equivalence relation $E$, denote by $A(\Gamma, E)=A(\Gamma,[E])$ the subspace of $A(\Gamma, X, \mu)$ consisting of all $a \in A(\Gamma, X, \mu)$ "contained" in $E$, in the sense that $\forall \gamma \in \Gamma\left(\gamma^{a} \in[E]\right)$, (see $[\mathbf{K} \mathbf{1}$, Section 6$\left.]\right)$. Then $A(\Gamma, E)$ is separable and closed in $(A(\Gamma, X, \mu), u)$, so a Polish space in the uniform topology.

Consider now the case $\Gamma=\mathbb{F}_{\infty}$, the free group with a countably infinite sequence of free generators $\left(\gamma_{n}\right)$. Then a complete compatible metric for $(A(\Gamma, E), u)$ is given by

$$
\delta\left(a_{1}, a_{2}\right)=\sum_{n=0}^{\infty} 2^{-(n+1)} d_{u}\left(\gamma_{n}^{a_{1}}, \gamma_{n}^{a_{2}}\right) .
$$

Fix a sequence of Borel involutions $\left(T_{n}\right)$ that generates $E$, i.e.,

$$
x E y \Longleftrightarrow \exists n\left(T_{n}(x)=y\right),
$$

and define the following complete metric on $S(E)$

$$
\rho\left(F_{1}, F_{2}\right)=\sum_{n=0}^{\infty} 2^{-(n+1)} \mu\left(A_{T_{n}, F_{1}} \Delta A_{T_{n}, F_{2}}\right)
$$

(see Section 2.2).

We define a map $\Psi: S(E) \rightarrow A\left(\mathbb{F}_{\infty}, E\right)$ as follows: We let $\Psi(F)=a$, where the action $a$ is defined by letting $\gamma_{i}^{a}(x)=T_{i}(x)$, if $T_{i}(x) F x$, and $\gamma_{i}^{a}(x)=x$, otherwise. Then $F=E_{a}=E_{\Psi(F)}$.

Proposition 2.7. $\Psi$ is an isometric embedding of $(S(E), \rho)$ onto a closed subspace of $\left(A\left(\mathbb{F}_{\infty}, E\right), \delta\right)$.

Therefore $S(E)$ can be identified with a closed subspace of $\left(A\left(\mathbb{F}_{\infty}, E\right), u\right)$ and inherits a Polish topology from this identification.

2.7. Equivalence of the topologies. We have described a number of topologies for $S(E)$ in the previous subsections. They all turn out to be the same:

Theorem 2.8. The topologies on $S(E)$ given in Sections 2.1-2.6 are identical. 
From now on will call this the topology of $S(E)$. Note that $S(E)$ is contractible (to the equality relation) by the map $\varphi: S(E) \times[0,1] \rightarrow S(E)$ given by $\varphi(F, t)=$ $F \mid[1,1-t] \cup\{(x, x): x \in(1-t, 1]\}$, where without loss of generality we assume that $X=[0,1]$ and $\mu$ is Lebesgue measure.

There is also another topology on $S(E)$, which we call the uniform topology on $S(E)$. The uniform topology contains the topology of $S(E)$ but it is not separable, when $E$ is aperiodic, i.e., all its classes are infinite. The uniform topology is induced by the following equivalent complete metrics:

$$
\begin{gathered}
\tau_{\infty}\left(F_{1}, F_{2}\right)=\sup _{T \in[E]} \mu\left(A_{T, F_{1}} \Delta A_{T, F_{2}}\right), \\
\tau_{\infty}^{\prime}\left(F_{1}, F_{2}\right)=\sup _{T \in[E]}\left|\mu\left(A_{T, F_{1}}\right)-\mu\left(A_{T, F_{2}}\right)\right| .
\end{gathered}
$$

2.8. Limits of sequences. Itv is easy to see that if $F_{0} \subseteq F_{1} \subseteq F_{2} \subseteq \ldots$ is an increasing sequence in $S(E)$, then $F_{n} \rightarrow \bigcup_{n} F_{n}$ and if $F_{0} \supseteq F_{1} \supseteq F_{2} \supseteq \ldots$ is a decreasing sequence in $S(E)$, then $F_{n} \rightarrow \bigcap_{n} F_{n}$, in the topology of $S(E)$.

The next result shows the relationship of a converging sequence to its limit in the topology of $S(E)$.

Theorem 2.9. Let $F_{n}, F \in S(E)$ and $F_{n} \rightarrow F$. Then for each $i$, there is an increasing sequence $n_{0}^{(i)}<n_{1}^{(i)}<\ldots$, so that $\left(n_{m}^{(i+1)}\right)_{m \in \mathbb{N}}$ is a subsequence of $\left(n_{m}^{(i)}\right)_{m \in \mathbb{N}}$ and

$$
F=\bigcup \bigcap_{m \geq m} F_{n_{k}^{(m)}}
$$

There is also an analog for the uniform topology:

Theorem 2.10. Let $F_{n}, F \in S(E)$ and $F_{n} \rightarrow F$ in the uniform topology. Then there is an increasing sequence $n_{0}<n_{1}<\ldots$, so that

$$
F=\bigcup \bigcap_{m \geq m} F_{n_{k}}
$$

Theorem 2.9 can be used to characterize the closure of certain subsets of $S(E)$. For $\mathcal{R}$ a class of measure preserving $\mathrm{CBER}$ on $(X, \mu)$, let

$$
\mathcal{R}_{\downarrow}=\left\{\bigcap_{n} F_{n}: F_{0} \supseteq F_{1} \supseteq \ldots, F_{n} \in \mathcal{R}\right\},
$$

and

$$
\mathcal{R}_{\uparrow}=\left\{\bigcup_{n} F_{n}: F_{0} \subseteq F_{1} \subseteq \ldots, F_{n} \in \mathcal{R}\right\} .
$$

Then we have, letting for for any class $\mathcal{R}$ of measure preserving CBER:

$$
\mathcal{R}^{*}=\left(\mathcal{R}_{\downarrow}\right) \uparrow .
$$

Theorem 2.11. Let $\mathcal{R} \subseteq S(E)$ be closed under finite intersections. Then

$$
\overline{\mathcal{R}}=\mathcal{R}^{*}
$$

(where $\overline{\mathcal{R}}$ is the closure of $\mathcal{R}$ in $S(E)$ ). 
It follows that for any class $\mathcal{R}$ of measure preserving CBER (not necessarily contained in some $S(E)$ ) closed under finite intersections:

$$
\left(\mathcal{R}^{*}\right)^{*}=\mathcal{R}^{*} \text {. }
$$

A class $\mathcal{R}$ of measure preserving CBER is hereditary if $F_{1} \subseteq F_{2} \in \mathcal{R} \Longrightarrow F_{1} \in$ $\mathcal{R}$. Theorem 2.11 also implies the following result concerning arbitrary hereditary classes of equivalence relations (not necessarily contained in some $S(E)$ ). It was originally proved (in a somewhat stronger form not requiring invariance of the measure) in Boykin-Jackson [BJ, page 116].

Corollary 2.12 (Boykin-Jackson $[\mathbf{B J}]$ ). Let $\mathcal{R}$ be a hereditary class of measure preserving CBER. Then $\mathcal{R}_{\uparrow}$ is closed under taking unions of increasing sequences of relations, i.e., $\left(\mathcal{R}_{\uparrow}\right)_{\uparrow}=\mathcal{R}_{\uparrow}$.

Put also

$$
\mathcal{R}_{*}=\left(\mathcal{R}_{\uparrow}\right)_{\downarrow} .
$$

If $\mathcal{R}$ is closed under finite intersections, then it follows from Theorem 2.11 that $\mathcal{R}_{*} \subseteq \mathcal{R}^{*}$.

Problem 2.13. If $\mathcal{R}$ is closed under finite intersections, is it true that $\mathcal{R}_{*}=\mathcal{R}^{*}$ ?

\section{Coherence of Topologies}

3.1. The weak topology on $\mathcal{E}$. We consider now the relation of the topologies of $S(E), S(F)$, when $E \subseteq F$.

Theorem 3.1. Let $E \subseteq F$. Then $S(E)$ is a closed subset of $S(F)$ and the topology of $S(E)$ is the relative topology from $S(F)$.

Recall that $\mathcal{E}$ is the set of all measure preserving $\operatorname{CBER}$ on $(X, \mu)$ (where we identify two equivalence relations if they agree a.e.). Thus $\mathcal{E}=\bigcup_{E \in \mathcal{E}} S(E)$. By the preceding Theorem 3.1, the topologies on $S(E), S(F)$ agree on $S(E) \cap S(F)=$ $S(E \cap F)$ and $S(E \cap F)$ is closed in $S(E)$ and $S(F)$. So we can define the weak topology on $\mathcal{E}$ induced by the spaces $S(E)$, which is the topology defined by declaring that $U \subseteq \mathcal{E}$ is open iff $U \cap S(E)$ is open in $S(E)$ for all $E \in \mathcal{E}$. In particular $f: \mathcal{E} \rightarrow Y, Y$ a topological space, is continuous if $f \mid S(E): S(E) \rightarrow Y$ is continuous for all $E \in \mathcal{E}$. Also on each $S(E)$ the relative topology from $\mathcal{E}$ coincides with its topology and $S(E)$ is closed in $\mathcal{E}$. (For the general concept of weak topology on a set induced by topologies on families of subsets, see, e.g., [D, VI.8].)

We should also note here that for $E \subseteq F, S(E)$ is a retract of $S(F)$, with the retraction given by the map $S(F) \ni R \mapsto R \cap E \in S(E)$. From this it follows that the map $S(F) \ni E \mapsto S(E) \in \mathcal{F}^{*}(S(F))$, where $\mathcal{F}^{*}(S(F))$ is the space of all nonempty closed subsets of $S(F)$ equipped with the Effros Borel structure, is a Borel map.

3.2. Properties of the weak topology. We will next give another description of the weak topology of $\mathcal{E}$. Consider the compact space $[0,1]^{\operatorname{Aut}(X, \mu)}$ with the product topology. Define

$$
\Pi: \mathcal{E} \rightarrow[0,1]^{\operatorname{Aut}(X, \mu)}
$$

by

$$
\Pi(F)(T)=d(T, F)=\mu(\{x:(x, T(x)) \notin F\}) .
$$


Since $[F]=\{T: d(T, F)=0\}$, clearly $\Pi$ is injective.

Theorem 3.2. The map $\Pi$ is a homeomorphism of $\mathcal{E}$ into $[0,1]^{\operatorname{Aut}(X, \mu)}$.

Thus $\mathcal{E}$ can be viewed as a subspace of $[0,1]^{\operatorname{Aut}(X, \mu)}$, so, in particular, it is Hausdorff. On the other hand it is neither separable or first countable.

Proposition 3.3. The weak topology on $\mathcal{E}$ is not separable and not first countable.

In fact we will see in the next section that $\mathcal{E}$ does not admit any "definable" separable, metrizable topology.

\section{Relations with the Space of Actions}

4.1. Parametrization by actions. Denote by $(A(\Gamma, X, \mu), w)$ the space of measure preserving actions of $\Gamma$ on $(X, \mu)$ with the weak topology $w$ (see $[\mathbf{K}$, Section $10,(\mathbf{A})])$. Here we consider the product topology on $\operatorname{Aut}(X, \mu)^{\Gamma}$, where $\operatorname{Aut}(X, \mu)$ is given the weak topology. The space $A(\Gamma, X, \mu)$ is then viewed as a closed subspace of $\operatorname{Aut}(X, \mu)^{\Gamma}$ in this product topology.

To see another aspect of the global structure of $\mathcal{E}$, consider the Polish space $\left(A\left(\mathbb{F}_{\infty}, X, \mu\right), w\right)$. The map $a \mapsto E_{a}$ is a surjection from $A\left(\mathbb{F}_{\infty}, X, \mu\right)$ to $\mathcal{E}$ and provides a canonical parametrization of $\mathcal{E}$. Let

$$
a \sim_{\mathbb{F}_{\infty}} b \Longleftrightarrow E_{a}=E_{b}
$$

be the associated equivalence relation, so that $\mathcal{E}=A\left(\mathbb{F}_{\infty}, X, \mu\right) / \sim_{\mathbb{F}_{\infty}}$.

Proposition 4.1. $\sim_{\mathbb{F}_{\infty}}$ is $F_{\sigma \delta}$.

Below let $E_{\text {ctble }}$ be the equivalence relation on $P^{\mathbb{N}}$, where $P$ is an uncountable Polish space, given by

$$
\left(x_{n}\right) E_{\text {ctble }}\left(y_{n}\right) \Longleftrightarrow\left\{x_{n}: n \in \mathbb{N}\right\}=\left\{y_{n}: n \in \mathbb{N}\right\} .
$$

Recall that a Borel equivalence relation $E$ on a standard Borel space $X$ is called smooth if there is a Borel map $f: X \rightarrow Y$, from $X$ to some standard Borel space $Y$, such that $x E y \Longleftrightarrow f(x)=f(y)$. It is well known that $E_{\text {ctble }}$ is a non-smooth equivalence relation and moreover it is $F_{\sigma \delta}$-complete (as a set of pairs). Below for Borel equivalence relations $E, F$ in Polish spaces $X, Y$, we let $E \leq_{c} F$ mean that there is a continuous reduction from $E$ to $F$, i.e., a continuous map $f: X \rightarrow Y$ with $x E y \Longleftrightarrow f(x) F f(y)$.

Theorem 4.2. $E_{\text {ctble }} \leq_{c} \sim_{\mathbb{F}_{\infty}}$, so, in particular, $\sim_{\mathbb{F}_{\infty}}$ is $F_{\sigma \delta}$-complete (as a set of pairs) and non-smooth.

It can be also shown that $\sim_{\mathbb{F}_{\infty}}$ is Borel reducible to an equivalence relation induced by a Borel action of a Polish group. In fact, by using a slightly different parametrization of $\mathcal{E}$, the associated equivalence relation is again $F_{\sigma \delta}$ and induced by a continuous action of a Polish group (see Törnquist [T, page 33]).

The preceding show that it is not possible to find a "definable" injection of $\mathcal{E}$ into a standard Borel space, so in particular $\mathcal{E}$ does not admit any "definable" separable metrizable topology. The following remain open: 
Problem 4.3. What is the complexity of the equivalence relation (as a set of pairs) on the space $(A(\Gamma, X, \mu), w)$ given by

$$
a \sim_{\Gamma} b \Longleftrightarrow E_{a}=E_{b},
$$

for other groups $\Gamma$, e.g., $\Gamma=\mathbb{Z}$ ?

Problem 4.4. Determine the complexity of the equivalence relation $\sim_{\mathbb{F}_{\infty}}$ in the hierarchy of Borel equivalence relations under Borel reducibility.

4.2. Continuity of parametrization. Let now $\Gamma$ be an arbitrary countable group and consider again $(A(\Gamma, X, \mu), u)$ the space of measure preserving actions of $\Gamma$ on $(X, \mu)$ with the uniform topology and its closed subspace $A(\Gamma, E)$. We also let $E_{T_{1}, T_{2}}, \ldots$ be the equivalence relation induced by $T_{1}, T_{2}, \ldots$ in $\operatorname{Aut}(X, \mu)$.

Note that if $\Gamma=\mathbb{F}_{\infty}$ the map $a \mapsto E_{a}$ gives a parametrization of $S(E)$ by $A\left(\mathbb{F}_{\infty}, E\right)$, i.e., a surjective map from $A\left(\mathbb{F}_{\infty}, E\right)$ onto $S(E)$. We have the following selection result:

Theorem 4.5. There is a continuous map $\Psi: S(E) \rightarrow A\left(\mathbb{F}_{\infty}, E\right)$ such that for $F \in S(E), E_{\Psi(F)}=F$.

Concerning the complexity of the map $a \mapsto E_{a}$, we have:

Theorem 4.6. The map $A(\Gamma, E) \ni a \mapsto E_{a} \in S(E)$ is of Baire class 1 .

Corollary 4.7. Let $\Gamma=\mathbb{F}_{\infty}$. Let $\mathcal{P}$ be a property of equivalence relations such that

$$
\mathcal{P}_{E}^{*}=\left\{a \in A(\Gamma, E): E_{a} \in \mathcal{P}\right\}
$$

is Borel in $A(\Gamma, E)$. Then $\mathcal{P}_{E}=\mathcal{P} \cap S(E)$ is Borel in the topology of $S(E)$.

In particular, taking again $\Gamma=\mathbb{F}_{\infty}$, suppose $\mathcal{P}$ is a property of equivalence relations such that $\left\{a \in A(\Gamma, X, \mu): E_{a} \in \mathcal{P}\right\}$ is Borel in the weak topology of $A(\Gamma, X, \mu)$. Since this is contained in the uniform topology of $A(\Gamma, X, \mu)$, this set is Borel in the uniform topology of $A(\Gamma, X, \mu)$ and thus $\left\{a \in A(\Gamma, E): E_{a} \in \mathcal{P}\right\}$ is Borel in (the uniform topology of) $A(\Gamma, E)$. Therefore $\mathcal{P}_{E}$ is Borel in $S(E)$.

Problem 4.8. For which countable groups $\Gamma$ is the map $A(\Gamma, E) \ni a \mapsto E_{a} \in S(E)$ continuous, for each $E$ ?

A.Tserunyan and R. Tucker-Drob found the first examples that showed that this map is not always continuous. It turned out now that we have the following general fact. Recall that a measure preserving CBER is ergodic if every $E$-invariant Borel set is either null or co-null.

Theorem 4.9. Let $E$ be ergodic. Let $\Gamma$ be a countable infinite amenable group. Then the map $A(\Gamma, E) \ni a \mapsto E_{a} \in S(E)$ is not continuous.

We now define a stronger topology than the uniform topology on $A(\Gamma, X, \mu)$ (see $[\mathbf{K}$, Remark in page 103]). It is induced by the complete metric

$$
\delta_{\Gamma, \infty}(a, b)=\sup _{\gamma \in \Gamma} d_{u}\left(\gamma^{a}, \gamma^{b}\right) .
$$

Recall also the metric $\tau_{\infty}$ on $S(E)$ defined in Section 2.7. The main fact is that the map $a \in A(\Gamma, E) \mapsto E_{a} \in S(E)$ is Lipschitz in the metrics $\delta_{\Gamma, \infty}, \tau_{\infty}$. 
Theorem 4.10. For any countable group $\Gamma$ and any $a, b \in A(\Gamma, E)$,

$$
\tau_{\infty}\left(E_{a}, E_{b}\right) \leq 80 \delta_{\Gamma, \infty}(a, b) .
$$

In particular, $A(\Gamma, E) \ni a \mapsto E_{a} \in S(E)$ is continuous from the $\delta_{\Gamma, \infty}$-topology on $A(\Gamma, E)$ to the uniform topology of $S(E)$ (and thus to the topology of $S(E)$ ).

It is known that when $\Gamma$ has property $(\mathrm{T})$ the $\delta_{\Gamma, \infty}$-topology on $A(\Gamma, E)$ coincides with the (uniform) topology of $A(\Gamma, E)$ (see [K, Remark in page 103]), so we have the following result originally proved by R. Tucker-Drob:

Corollary 4.11 (Tucker-Drob). If a countable group $\Gamma$ has property $(\mathrm{T})$, then the map $A(\Gamma, E) \ni a \mapsto E_{a} \in S(E)$ is continuous.

In view of Theorem 4.9 and Corollary 4.11, one can consider the following more precise version of Problem 4.8.

Problem 4.12. Let $\Gamma$ be a countable group. Is it true that the map $A(\Gamma, E) \ni a \mapsto$ $E_{a} \in S(E)$ is continuous for every $E$ iff the group $\Gamma$ has property $(\mathrm{T})$ ?

\section{The Action of the Automorphism Group}

Let $E$ be an aperiodic, measure preserving CBER. We denote by $N(E)$ the group of measure preserving automorphisms of $E$, i.e., the group of all $T \in \operatorname{Aut}(X, \mu)$ such that $x E y \Longleftrightarrow T(x) E T(y)$, for all $x, y$ in a conull set. This can be identified with a closed subgroup of the isometry group of $\left([E], d_{u}\right)$ (see $[\mathbf{K}$, Section 6$]$ ), so it is a Polish group in the pointwise convergence topology. The group $[E]$ is a Polishable subgroup of $N(E)$, with the corresponding Polish topology being the uniform topology on $[E]$. The group $N(E)$ acts in the obvious way on $S(E)$ and it turns out that this action is continuous. The same holds for the restriction of this action to $[E]$ (equipped with the uniform topology). Since this action has $E$ as a fixed point, it is not minimal. The next question is whether it is topologically transitive, i.e., has a dense orbit.

Problem 5.1. Let $E$ be an aperiodic, measure preserving CBER. Is there a dense orbit for the action of $[E]$ on $S(E)$ ? Similarly for the action of $N(E)$.

The answer depends on the ergodic theoretic properties of $E$. This follows from the following result of $\mathrm{F}$. Le Maître.

Theorem 5.2 (Le Maître [LeM1]). The answer to Problem 5.1 is positive, when $E$ is ergodic, hyperfinite but negative, even for $N(E)$, if $E$ is generated by a measure preserving action of an infinite, countable group with property $(\mathrm{T})$.

Recall that $E$ is hyperfinite if it is the union of an increasing sequence of finite CBER (i.e., having all classes finite).

\section{Continuity of Operations}

We discuss here the continuity (or lack thereof) of various operations in $S(E)$.

Proposition 6.1. (i) The operation $\left(F_{1}, F_{2}\right) \mapsto F_{1} \cap F_{2}$ from $S(E) \times S(E)$ to $S(E)$ is continuous.

(ii) The map $\left(F_{1}, F_{2}\right) \mapsto F_{1} \times F_{2}$ from $S\left(E_{1}\right) \times S\left(E_{2}\right)$ to $S\left(E_{1} \times E_{2}\right)$ is continuous. 
(iii) The map

$$
\left(S(E) \times \mathrm{MALG}_{\mu}\right) \ni(F, A) \mapsto F \mid A \in S(E),
$$

where $F \mid A=\{(x, y):(x, y \in A \& x E y) \vee x=y\}$, is continuous.

(iv) The operation $\left(F_{1}, F_{2}\right) \mapsto F_{1} \vee F_{2}$ from $S(E) \times S(E)$ to $S(E)$ is not continuous, if $E$ is aperiodic. (Here $F_{1} \vee F_{2}$ is the smallest equivalence relation containing both $F_{1}, F_{2}$.) On the other hand, $\left(F_{1}, F_{2}\right) \mapsto F_{1} \vee F_{2}$ from $S(E) \times S(E)$ to $S(E)$ is of Baire class 1.

(v) The relations $F_{1} \subseteq F_{2}$ and $F_{1} \perp F_{2}$ (see [KM, Section 27]) are closed in $S(E) \times S(E)$

\section{Complexity Calculations}

7.1. Aperiodicity, finiteness and hyperfiniteness. We next discuss the complexity of various classes of equivalence relations. For any class $\mathcal{R}$ of measure preserving CBER and any given such relation $E$, we denote by

$$
\mathcal{R}_{E}=\mathcal{R} \cap S(E)
$$

the set of subequivalence relations of $E$ that are in the class $\mathcal{R}$. In particular $\mathcal{E}_{E}=S(E)$. Recall that an equivalence relation is finite if all its equivalence classes are finite and hyperfinite if it is the union of an increasing sequence of finite equivalence relations. As a consequence of Theorem 2.11 we have

Theorem 7.1. Let $\mathcal{H}$ be the class of hyperfinite equivalence relations. Then $\mathcal{H}_{E}$ is closed in $S(E)$.

Denote by $\mathcal{F}$, resp., $\mathcal{B F}$ the classes of equivalence relations which are finite, resp., bounded finite (i.e., for some $N$ each equivalence class has at most $N$ elements). It follows that

$$
\overline{\mathcal{F}_{E}}=\overline{\mathcal{B F}_{E}}=\mathcal{H}_{E} .
$$

In particular, $E$ is hyperfinite iff $\mathcal{F}_{E}$ is dense in $S(E)$ iff $\mathcal{B F}_{E}$ is dense in $S(E)$. It also follows from this that the map $S(F) \ni E \mapsto \mathcal{H}_{E} \in \mathcal{F}^{*}(S(F)$ ) is Borel (when $\mathcal{F}^{*}(S(F))$ is equipped with the Effros Borel structure).

Theorem 7.2. For every aperiodic $E, \mathcal{B F}_{E}$ is in $F_{\sigma}$ but not in $G_{\delta}$ in the topology of $S(E)$.

Theorem 7.3. The set $\mathcal{F}_{E}$ of finite equivalence relations in $S(E)$ is $F_{\sigma \delta}$ in the topology of $S(E)$.

Problem 7.4. If $E$ is aperiodic, is $\mathcal{F}_{E}$ not in $G_{\delta \sigma}$ for the topology of $S(E)$ ?

The following provides an affirmative answer when $E$ is ergodic.

Theorem 7.5. If $E$ is ergodic, then $\mathcal{F}_{E}$ is in $F_{\sigma \delta} \backslash G_{\delta \sigma}$ in the topology of $S(E)$.

Finally we calculate the complexity of the class of aperiodic (i.e., having infinite classes) equivalence relations.

Theorem 7.6. Let $\mathcal{A}$ be the class of aperiodic equivalence relations. Then $\mathcal{A}_{E}$ is a $G_{\delta}$ set in the topology of $S(E)$. Moreover, if $E$ is aperiodic, then $\mathcal{A}_{E}$ is dense and also in $G_{\delta} \backslash F_{\sigma}$. 
7.2. Finite and infinite index subrelations. Denote by FinIndex(E) (resp., InfIndex $(E))$ the set of all $F \in S(E)$ such that $[E: F]<\infty$, i.e., every $E$ class contains only finitely many $F$-classes (resp., $[E: F]=\infty$, i.e., every $E$-class contains infinitely many $F$-classes).

Proposition 7.7. The set InfIndex $(E)$ is $G_{\delta}$ in $S(E)$ and it is dense if $E$ is aperiodic.

Proposition 7.8. If $E$ is aperiodic, hyperfinite, then the set InfIndex $(E)$ is in $G_{\delta} \backslash F_{\sigma}$.

Problem 7.9. Let $E$ be aperiodic. Is InfIndex $(E)$ in $G_{\delta} \backslash F_{\sigma}$ ?

Proposition 7.10. The set FinIndex $(E)$ is $F_{\sigma \delta}$ in $S(E)$. If $E$ is aperiodic, hyperfinite, then it is also dense.

It turns out that one can find some aperiodic, hyperfinite $E$ such that FinIndex $(E)$ is in $F_{\sigma \delta} \backslash G_{\delta \sigma}$.

Problem 7.11. Assume $E$ is aperiodic. Is the set FinIndex $(E)$ in $F_{\sigma \delta} \backslash G_{\delta \sigma}$ ?

The set FinIndex $(E)$ is not always dense in $S(E)$.

Theorem 7.12. There is an ergodic E such that FinIndex $(E)$ is not dense in $S(E)$.

Problem 7.13. For what ergodic $E$ is FinIndex $(E)$ dense in $S(E)$ ?

Remark 7.14. In Vaes [Va] and Bowen [Bo1] examples are given of ergodic equivalence relations that do not have proper finite index ergodic subequivalence relations or proper finite index extensions.

Remark 7.15. In Popa [Po, Section 6.6] it is suggested that it might be possible that the cocycle superrigidity proved in that paper could be extended to target groups that are closed subgroups of the (infinitary) unitary group $U(H)$. From the proof of Theorem 7.12 one can see however that this fails for the infinite symmetric group $S_{\infty}$, which is a closed subgroup of $U(H)$.

7.3. Ergodic and strongly ergodic equivalence relations. We first calculate the complexity of the set of ergodic equivalence relations in $S(E)$. We denote by $\mathcal{E R \mathcal { G }}$ the class of measure preserving CBER which are ergodic.

Theorem 7.16. The set $\mathcal{E} \mathcal{R} \mathcal{G}_{E}$ of ergodic equivalence relations in $S(E)$ is $G_{\delta}$ in $S(E)$.

In the next section we will consider the question of density of $\mathcal{E} \mathcal{R} \mathcal{G}_{E}$ in $S(E)$.

An equivalence relation $F$ is called strongly ergodic or $\boldsymbol{E}_{0}$-ergodic iff for any Borel homomorphism $\pi: X \rightarrow Y$ from $F$ to a hyperfinite equivalence relation $R$ on $Y$ (i.e., $\left.x F x^{\prime} \Longrightarrow \pi(x) R \pi\left(x^{\prime}\right)\right)$, there is $y \in Y$ such that $\pi^{-1}\left([y]_{F}\right)$ has measure 1. By a result of Jones-Schmidt $[\mathbf{J S}]$ this is equivalent to the non-existence of non-trivial almost invariant sets for $F$ (see, e.g., [HK, Theorem A2.2], in which the hypothesis of ergodicity is unnecessary). We denote the class of all measure preserving CBER that are strongly ergodic by $\mathcal{E}_{0} \mathcal{R} \mathcal{G}$. For further reference, we call an action $a \in A(\Gamma, X, \mu)$ ergodic or strongly ergodic if $E_{a}$ has this property. 
We call an equivalence relation $F$ anti- $\boldsymbol{E}_{0}$-ergodic if there is homomorphism $\pi$ as above to a hyperfinite equivalence relation for which all preimages of $F$-classes are null. Denote by $\mathcal{A} \mathcal{E}_{0} \mathcal{R} \mathcal{G}$ the class of all anti- $E_{0}$-ergodic equivalence relations.

Proposition 7.17. The set $\mathcal{A E}_{0} \mathcal{R} \mathcal{G}_{E}$ is closed in $S(E)$.

Theorem 7.18. The set $\mathcal{E}_{0} \mathcal{R \mathcal { G }}_{E}$ is in the class $F_{\sigma} \cap G_{\delta}$ in $S(E)$.

Problem 7.19. Are there E for which Theorem 7.18 gives the optimal descriptive complexity of $\mathcal{E}_{0} \mathcal{R} \mathcal{G}_{E}$ ?

\section{Richly Ergodic Equivalence Relations}

We first note that for any $E, S(E) \backslash \mathcal{E} \mathcal{R} \mathcal{G}_{E}$ is dense in $S(E)$. This is because any $F \in S(E)$ can be approximated by equivalence relations of the form $F|(X \backslash A) \sqcup i d| A$, for Borel $A$ of small positive measure, which clearly are not ergodic. We will discuss here the problem of density for the ergodic subrelations. Note that $E$ is ergodic iff $\mathcal{E} \mathcal{R} \mathcal{G}_{E} \neq \emptyset$. Let us call an ergodic equivalence relation $E$ for which $\mathcal{E} \mathcal{R G}_{E}$ is dense in $S(E)$ richly ergodic.

Problem 8.1. Which among the ergodic equivalence relations $E$ are richly ergodic?

We will first see that in some sense most $E$ are richly ergodic. If $E_{0} \subseteq E_{1} \subseteq \ldots$ is an increasing sequence, we say that $\left(E_{n}\right)_{n \in \mathbb{N}}$ is strongly increasing if for each $n$ there is an ergodic $T \in\left[\bigcup_{n} E_{n}\right]$ such that $E_{n} \perp E_{T}$.

Proposition 8.2. If $\left(E_{n}\right)$ is strongly increasing and $E=\bigcup_{n} E_{n}$, then $E$ is richly ergodic.

Using this one can show the following:

Proposition 8.3. For any $E$, there is $E^{\prime} \supseteq E$ which is richly ergodic.

Proposition 8.4. If $E_{0} \subseteq E_{1} \subseteq \ldots$ are richly ergodic, so is $E=\bigcup_{n} E_{n}$.

Thus the collection of richly ergodic equivalence relations is "very large" in the sense that it is $\omega$-closed and cofinal in the class of all equivalence relations under $\subseteq$. We next discuss some particular classes of richly ergodic equivalence relations. Below let $\mathcal{E} \mathcal{R G} \mathcal{H}=\mathcal{E} \mathcal{R G} \cap \mathcal{H}$ be the class of ergodic, hyperfinite equivalence relations.

Theorem 8.5. For any ergodic $E, \mathcal{E R \mathcal { H }}_{E}$ is dense in $\mathcal{H}_{E}$. In particular, every hyperfinite ergodic equivalence relation is richly ergodic.

Another way to generate richly ergodic equivalence relations is the following:

Proposition 8.6. Let $\Gamma=\Gamma_{1} * \Gamma_{2} * \cdots$, where each countable group $\Gamma_{n}$ is nontrivial. Let $E$ be induced by a free, measure preserving, mixing action of $\Gamma$. Then $E$ is richly ergodic.

We next see that there exist ergodic but not richly ergodic equivalence relations. These arise in the context of the so-called non-approximable equivalence relations, introduced in the paper Gaboriau-Tucker-Drob $[\mathbf{G T}]$. Let $E$ be a measure preserving CBER on $(X, \mu)$. We say that $E$ is non-approximable if whenever $E=\bigcup_{n} F_{n}$, where $F_{n}$ are Borel equivalence relations with $F_{0} \subseteq F_{1} \subseteq F_{2} \ldots$, then there is $n$ 
and a positive measure Borel set $A$ with $E\left|A=F_{n}\right| A$. It is an unpublished result of Gaboriau that if $a \in A(\Gamma, X, \mu)$, where $\Gamma$ is an infinite property (T) group, and $a$ is ergodic, then $E_{a}$ is non-approximable. This can be also seen as an application of [IKT, Corollary 5.4 and Corollary 2.15]. In [GT] the authors also show that if $a \in A(\Gamma \times \Delta, X, \mu)$ is a free action, where $\Gamma, \Delta$ are finitely generated, and $a \mid \Gamma$ is strongly ergodic while $a \mid \Delta$ is ergodic, then $E_{a}$ is non-approximable. We now have:

Theorem 8.7. If $E$ is ergodic and non-approximable, then $E$ is not richly ergodic.

Note that $E$ is $E_{0}$-ergodic iff $\mathcal{E}_{0} \mathcal{R} \mathcal{G}_{E} \neq \emptyset$, Finally we call an equivalence relation $E$ richly $\boldsymbol{E}_{0}$-ergodic if $\mathcal{E}_{0} \mathcal{R \mathcal { G }}_{E}$ is dense in $S(E)$. By Theorem 8.7 and the preceding paragraph, it clearly follows that there are $E_{0}$-ergodic equivalence relations which are not richly $E_{0}$-ergodic. There are also richly $E_{0}$-ergodic equivalence relations,

Problem 8.8. Which among the $E_{0}$-ergodic equivalence relations $E$ are richly $E_{0^{-}}$ ergodic?

\section{The Cost Function}

Let $F$ be a measure preserving CBER and $G \subseteq F$ a (simple, undirected) graph contained in $F$. Let $C_{\mu}(G)=\frac{1}{2} M(G)$, where the measure $M$ on $F$ is defined as in Section 2.3. We say that $G$ is a graphing of $F$ if $F$ is the equivalence relation generated by $G$ (i.e., the $F$-classes are the connected components of $G$ ). Finally define the cost of $F$ by

$$
C(F)=C_{\mu}(F)=\inf \left\{C_{\mu}(G): G \text { is a graphing of } F\right\} .
$$

Also for $a \in A(\Gamma, X, \mu)$, let $C(a)=C\left(E_{a}\right)$. For an exposition of the theory of cost see, for example, $[\mathbf{K M}]$. We will discuss here the complexity of the function $S(E) \ni F \mapsto C(F) \in[0, \infty]$.

Let

$$
\begin{aligned}
& \text { FinCost }_{E}=\{F \in S(E): C(F)<\infty\}, \\
& \text { InfCost }_{E}=\{F \in S(E): C(F)=\infty\} .
\end{aligned}
$$

Proposition 9.1. The set FinCost F $_{\text {is }}$ dense in $S(E)$.

We next have the following dichotomy:

Theorem 9.2. For any aperiodic measure preserving CBER E, exactly one of the following holds:

(i) For every $F \in S(E), C(F) \leq 1$,

(ii) InfCost $_{E}$ is dense in the uniform topology of $S(E)$.

Remark 9.3. It is unknown if condition (i) in Theorem 9.2 is equivalent to hyperfiniteness.

The following problem is open. For convenience, we will say that $E$ is of type II if it is aperiodic and there is $F \in S(E)$ with $C(F)>1$.

Problem 9.4. Let $E$ be a type II equivalence relation. Is InfCost $_{E}$ comeager in $S(E)$ ?

We will next consider the descriptive complexity of the cost function. 
Proposition 9.5. The set FinCost F $_{E}$ is analytic in $S(E)$ and the cost function $F \mapsto C(F)$ is Borel on FinCost . $_{\text {. }}$

The following is an open problem:

Problem 9.6. Is the cost function $F \mapsto C(F)$ Borel on $S(E)$ ? Equivalently is the

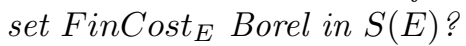

We next notice some related facts and questions. It is clear from Theorem 9.2 that for each $E$ of type II the sets $\{F \in S(E): C(F)>r\},\{F \in S(E): C(F) \geq r\}$, for $r \in \mathbb{R}, r>0$, are not uniformly closed. One can also see that for some $E$ the sets $\{F \in S(E): C(F)<r\}, r>1,\{F \in S(E): C(F) \leq r\}, r \geq 1$, are not closed. The following problem is open:

Problem 9.7. Are the sets

$$
\begin{aligned}
& \{F \in S(E): C(F)<r\}, r>1, \\
& \{F \in S(E): C(F)<\infty\}, \\
& \{F \in S(E): C(F) \leq r\}, r \geq 1 .
\end{aligned}
$$

uniformly closed?

One can also use these observations to answer a question that arises from $[\mathbf{K}$, First Remark in page 78]. It is shown there that when the infinite group $\Gamma$ is finitely generated, the cost function $C$ on $A(\Gamma, E)$ is upper semicontinuous. Is that true for arbitrary infinite $\Gamma$ ? The answer is negative:

Proposition 9.8. For any equivalence relation $E$ of type II, the function $A\left(\mathbb{F}_{\infty}, E\right) \ni$ $a \mapsto C(a)$ is not upper semicontinuous.

Using the theory of cost on can also see that the analog of Theorem 4.5 fails for $\mathbb{F}_{n}, n \geq 2$. Below let $\mathcal{F}_{n, E}=\left\{F \in S(E): \exists a \in A\left(\mathbb{F}_{n}, E\right)\left[E_{a}=E\right]\right\}$.

Proposition 9.9. Let $n \geq 2$. If $E$ is of type II, there is no continuous function $\Psi_{n}: \mathcal{F}_{n, E} \rightarrow A\left(\mathbb{F}_{n}, E\right)$ such that $E_{\Psi_{n}(F)}=F$.

Finally the set $\mathcal{F}_{n, E}$ is analytic in $S(E)$. The following problem is open:

Problem 9.10. Let $n \geq 2$. Is there a Borel function $\Psi_{n}: \mathcal{F}_{n, E} \rightarrow A\left(\mathbb{F}_{n}, E\right)$ such that $E_{\Psi_{n}(F)}=F$ ?

For $n=1, \mathcal{F}_{1, E}=\mathcal{H}_{E}$, thus, by Theorem 7.1, $\mathcal{F}_{1, E}$ is closed in $S(E)$ and we will see in Theorem 11.1 that Problem 9.10 has a positive solution for $n=1$. (Note that $A\left(\mathbb{F}_{1}, E\right)=A(\mathbb{Z}, E)$ is homeomorphic to $[E]$.) However we do not know if there is continuous $\Psi_{1}: \mathcal{F}_{1, E} \rightarrow A(\mathbb{Z}, E)$ with $E_{\Psi_{1}(F)}=F$.

\section{Normality}

We next discus normal subequivalence relations, see $[\mathbf{F S Z}]$. Let $E$ be ergodic and let $N=[E: F] \leq \infty$ be the index of $F$ in $E$, i.e., the number of $F$-classes in each $E$-class. A sequence $\left(\varphi_{n}\right)_{n<N}$ of Borel functions on $X$ such that for each $x,\left(\left[\varphi_{n}(x)\right]_{F}\right)_{n<N}$ is an injective enumeration of the $F$-classes in $[x]_{E}$ is called a choice sequence. Again we identify two such sequences if they agree a.e. Every $F$ admits a choice sequence and if $F$ is also ergodic, then such $\left(\varphi_{n}\right)_{n<N}$ can be found which are in $\operatorname{Aut}(X, \mu)$ (see [FSZ, Lemma 1.3]). 
Let $E$ be ergodic. A subequivalence relation $F \in S(E)$ is normal in $E$, in symbols

$$
F \triangleleft E,
$$

if there are choice sequences which are $F$-invariant. In particular, if $F \triangleleft E$ and $F$ is ergodic, then one can find choice sequences which are $F$-invariant and in $\operatorname{Aut}(X, \mu)$. We now have the following result concerning the complexity of the set of normal subequivalence relations.

Theorem 10.1. The set $N$ ormal $(E)$ of normal subequivalence relations of an ergodic equivalence relation $E$ is Borel in $S(E)$.

Problem 10.2. What is the Borel complexity of Normal(E) in $S(E)$ ?

\section{A Selection Theorem for Hyperfiniteness}

Recall that $\mathcal{H}$ is the class of hyperfinite equivalence relations. For each $E$, the set $\mathcal{H}_{E}$ is closed in $S(E)$ by Theorem 7.1. Also the set $\mathcal{E} \mathcal{R} \mathcal{G} \mathcal{H}_{E}$ of ergodic hyperfinite subequivalence relations of $E$ is a $G_{\delta}$ set in $S(E)$ by Theorem 7.1 and Theorem 7.16.

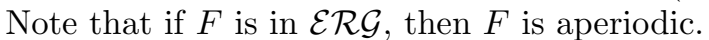

We now have the following selection result.

Theorem 11.1. There is a Borel function $\Theta: \mathcal{H}_{E} \rightarrow[E]$ such that for $F \in \mathcal{H}_{E}$, if $\Theta(F)=T$, then $F=E_{T}$ (i.e., $x F y \Longleftrightarrow \exists n \in \mathbb{Z}\left(T^{n}(x)=y\right)$ ).

It can be also shown that one can obtain in a Borel way witness to hyperfiniteness.

Theorem 11.2. There is a Borel function $H: \mathcal{H}_{E} \rightarrow S(E)^{\mathbb{N}}$ such that for $F \in \mathcal{H}_{E}$ we have that for each $n, H(F)_{n} \in \mathcal{B F}_{E}, H(F)_{n} \subseteq H(F)_{n+1}$, and $F=\bigcup_{n} H(F)_{n}$.

\section{Invariant, Random Equivalence Relations on Groups}

12.1. Equivalence relations on groups. For each countable group $\Gamma$, denote by $\operatorname{Eq}(\Gamma)$ the space of equivalence relations on $\Gamma$. This is a compact subspace of $2^{\Gamma^{2}}$. The group $\Gamma$ acts continuously by translation on $\operatorname{Eq}(\Gamma)$ : if $\gamma \in \Gamma, e \in \operatorname{Eq}(\Gamma)$, then

$$
(\delta, \epsilon) \in \gamma \cdot e \Longleftrightarrow\left(\gamma^{-1} \delta, \gamma^{-1} \epsilon\right) \in e
$$

Let $\sigma$ be a Borel probability measure on $\operatorname{Eq}(\Gamma)$. If $\sigma$ is invariant under the action of $\Gamma$, we say that $\sigma$ is an invariant, random equivalence relation (IRE) on $\Gamma$. We denote by $\operatorname{IRE}(\Gamma)$ the space of these measures.

Clearly $\operatorname{IRE}(\Gamma)$ is a compact subspace of the space of all Borel probability measures on $\operatorname{Eq}(\Gamma)$ (which is equipped, as usual, with the weak*-topology, in which it is compact metrizable)

There is a canonical connection between subequivalence relations of the equivalence relation $E_{a}$ induced by an action $a \in A(\Gamma, X, \mu)$ and IRE on $\Gamma$, which is a special case of structurability of such equivalence relations. See [KM, 29.1], [CK, Section 2], and [T-D, Appendix A] for the particular case of equivalence relations.

Let $a \in A(\Gamma, X, \mu)$ and put $E=E_{a}$. Given $F \in S(E)$, define the map

$$
e_{F}^{a}=e_{F}: X \rightarrow \operatorname{Eq}(\Gamma)
$$

by

$$
(\gamma, \delta) \in e_{F}(x) \Longleftrightarrow\left(\gamma^{-1} \cdot x, \delta^{-1} \cdot x\right) \in F
$$


Then $e_{F}$ is a $\Gamma$-equivariant Borel function. Put

$$
\sigma^{a}(F)=\sigma(F)=\left(e_{F}\right)_{*} \mu .
$$

Thus $\sigma^{a}(F) \in \operatorname{IRE}(\Gamma)$.

Proposition 12.1. The map $\sigma^{a}: S(E) \rightarrow \operatorname{IRE}(\Gamma)$ is continuous.

Remark 12.2. The map $\sigma^{a}$ is not injective. Consider, for example, the shift action $s$ of $\Gamma$ on $[0,1]^{\Gamma}$, with the usual product measure. Let $F_{1}=E_{s} \cap\{(x, y): x(1)=$ $y(1)\}, F_{2}=E_{s} \cap\{(x, y): x(\gamma)=y(\gamma)\}$, where $\gamma \neq 1$. Then $e_{F_{1}}=e_{F_{2}}$ is the constant function with value the equality relation $=_{\Gamma}$ on $\Gamma$, so $\sigma\left(F_{1}\right)=\sigma\left(F_{2}\right)$ is the Dirac measure at $=_{\Gamma}$ but $F_{1} \neq F_{2}$.

It turns out that every IRE is generated by the above procedure for some, in fact free, action $a$ and equivalence relation $F$. Below we denote by $\operatorname{FR}(\Gamma, X, \mu)$ the set of free actions in $A(\Gamma, X, \mu)$. An action is free if for $\gamma \neq 1, \gamma \cdot x \neq x$, $\mu$-a.e.

Proposition 12.3. IRE $(\Gamma)=\left\{\sigma^{a}(F): a \in A(\Gamma, X, \mu), F \in S\left(E_{a}\right)\right\}=\left\{\sigma^{a}(F): a \in\right.$ $\left.\operatorname{FR}(\Gamma, X, \mu), F \in S\left(E_{a}\right)\right\}$.

A special case of the above construction of IRE is the following. Let $Y$ be a standard Borel space and $F$ a Borel equivalence relation on $Y$. Consider the product space $X=Y^{\Gamma}$ with the shift action $s_{Y}$ of $\Gamma$ on this space and let $\mu$ be a shift-invariant probability measure on $X$. Define the equivalence relation $\tilde{F}$ on $X$ by $x \tilde{F} y \Longleftrightarrow x E_{s_{Y}} y \& x(1) F y(1)$. Let $e_{\tilde{F}}: X \rightarrow$ Eq $(\Gamma)$ be the associated map, so that $(\gamma, \delta) \in e_{\tilde{F}}(x) \Longleftrightarrow x(\gamma) F x(\delta)$. Finally consider the IRE $\sigma^{s_{X}}(\tilde{F})$.

Problem 12.4. Is every element of $\operatorname{IRE}(\Gamma)$ of the form $\sigma^{s_{X}}(\tilde{F})$, for some measure $\mu$ and Borel equivalence relation $F$ on $Y$ ? What if we take $F$ to be the equality relation on $Y$ ?

Another way to obtain IRE is the following. Let $\operatorname{Sg}(\Gamma)$ be the space of subgroups of $\Gamma$, which is a compact subspace of $2^{\Gamma}$ on which $\Gamma$ acts continuously by conjugation. An invariant, random subgroup (IRS) of $\Gamma$ is a conjugation invariant Borel probability measure on $\operatorname{Sg}(\Gamma)$. Denote the space of such measures by $\operatorname{IRS}(\Gamma)$. There is a canonical homeomorphism $\Sigma$ from $\operatorname{Sg}(\Gamma)$ into $\operatorname{Eq}(\Gamma)$ given by $(\gamma, \delta) \in$ $\Sigma(H) \Longleftrightarrow \gamma \delta^{-1} \in H$. Thus the equivalence classes of $\Sigma(H)$ are the right cosets of $H$. The range of $\Sigma$ consists of the equivalence relations induced by the cosets of a subgroup of $\Gamma$. The embedding $\Sigma$ is also $\Gamma$-equivariant, thus if $\mu \in \operatorname{IRS}(\Gamma)$, then $\Sigma_{*} \mu \in \operatorname{IRE}(\Gamma)$ and the range of $\Sigma_{*}$ consists of the IRE that concentrate on the range of $\Sigma$. This forms a proper compact subset of $\operatorname{IRE}(\Gamma)$. Tucker-Drob [T-D, Appendix A] characterizes $\Sigma_{*}(\operatorname{IRS}(\Gamma))$ as consisting of exactly those $\sigma^{a}(F)$ for $F \subseteq E_{a}$ that are normalized by $a$, which means that each $\gamma^{a}$ is an automorphism of $F$, i.e., $x F y \Longleftrightarrow \gamma^{a}(x) F \gamma^{a}(y)$.

12.2. Classes of invariant, random equivalence relations. We say that $\sigma \in$ $\operatorname{IRE}(\Gamma)$ is an aperiodic IRE if it concentrates on the equivalence relations all of whose classes are infinite. It is an infinite index IRE if it concentrates on the equivalence relations that have infinitely many classes. Both the aperiodic and the infinite index IRE form $G_{\delta}$ sets in $\operatorname{IRE}(\Gamma)$. Similarly $\sigma$ is a finite index IRE if it concentrates on the equivalence relations that have only finitely many classes. 
Finally, $\sigma$ is a finite IRE if it concentrates on the equivalence relations all of whose classes are finite.

We now have the following results:

Theorem 12.5. Let $\Gamma$ be an infinite countable group. The generic IRE on $\Gamma$ is aperiodic and has infinite index.

Theorem 12.6. Let $\Gamma$ be an infinite amenable countable group. Then the finite index $\operatorname{IRE}$ are dense in $\operatorname{IRE}(\Gamma)$.

As in Problem 7.13, we do not know if this holds for all infinite $\Gamma$.

Theorem 12.7. Let $\Gamma$ be an infinite countable group. Then the following are equivalent:

(i) $\Gamma$ is amenable,

(ii) The finite $\operatorname{IRE}$ are dense in $\operatorname{IRE}(\Gamma)$, IRE.

(iii) The Dirac measure $\delta_{\Gamma \times \Gamma}$ on the equivalence relation $\Gamma \times \Gamma$ is a limit of finite

12.3. Bauer vs Poulsen. The space $\operatorname{IRE}(\Gamma)$ is a Choquet simplex (being the space of invariant Borel probability measures for a continuous action of $\Gamma$ on a compact metrizable space). Its extremal points are the ergodic IRE, whose set we denote by $\operatorname{ERGIRE}(\Gamma)$. We next consider the question of whether $\operatorname{IRE}(\Gamma)$ is a Bauer simplex, i.e., $\operatorname{ERGIRE}(\Gamma)$ is closed in $\operatorname{IRE}(\Gamma)$, or the Poulsen simplex, i.e., $\operatorname{ERGIRE}(\Gamma)$ is dense in $\operatorname{IRE}(\Gamma)$. By the results in Glasner-Weiss $[\mathbf{G W}]$, if $\Gamma$ has property $(\mathrm{T})$, then $\operatorname{IRE}(\Gamma)$ is a Bauer simplex. However the following is open:

Problem 12.8. Assume that the countable group $\Gamma$ does not have property $(\mathrm{T})$. Is $\operatorname{IRE}(\Gamma)$ the Poulsen simplex?

12.4. Weak containment and invariant, random equivalence relations. Recall that for $a, b \in A(\Gamma, X, \mu)$, we let $a \preceq b$ iff $a$ is weakly contained in $b$ (see $[\mathbf{K}]$, where $\prec$ is used instead of $\preceq$ ). Concerning the map $\sigma^{a}(F)$ that sends $F \in S\left(E_{a}\right), a \in A(\Gamma, X, \mu)$, to an IRE on $\Gamma$, we consider its "slice" corresponding to the $\preceq$-predecessors of an action $b$.

Theorem 12.9. Let $\Gamma$ be an infinite countable group and $b \in A(\Gamma, X, \mu)$. Then the set

$$
\left\{\sigma^{a}(F): a \in A(\Gamma, X, \mu), a \preceq b, F \in S\left(E_{a}\right)\right\}
$$

is a compact subset of $\operatorname{IRE}(\Gamma)$.

Corollary 12.10. Let $\Gamma$ be an infinite countable group and assume that $b \in$ $A(\Gamma, X, \mu)$ is ergodic but not strongly ergodic. Then the set

$$
\left\{\sigma^{a}(F): a \in A(\Gamma, X, \mu), a \preceq b, F \in S\left(E_{a}\right)\right\}
$$

is a compact convex subset of $\operatorname{IRE}(\Gamma)$.

\section{Ultraproducts of Equivalence Relations}

We will discuss here an ultraproduct construction for measure preserving CBER. One can find the definition of ultraproducts of measure spaces and actions in $[\mathbf{C K T}]$ but for the convenience of the reader we summarize it here. 
Let $\left(X_{n}, \mu_{n}\right), n \in \mathbb{N}$, be a sequence of standard probability spaces and denote by $\boldsymbol{B}\left(X_{n}\right)$ the $\sigma$-algebra of Borel sets of $X_{n}$. Let $\mathcal{U}$ be a non-principal ultrafilter on $\mathbb{N}$. For $P \subseteq \mathbb{N} \times X(X$ some set $)$ we write

$$
\mathcal{U} n P(n, x) \Leftrightarrow\{n: P(n, x)\} \in \mathcal{U} .
$$

On $\prod_{n} X_{n}$ define the equivalence relation

$$
\left(x_{n}\right) \sim_{\mathcal{U}}\left(y_{n}\right) \Leftrightarrow \mathcal{U} n\left(x_{n}=y_{n}\right),
$$

let $\left[\left(x_{n}\right)\right]_{\mathcal{U}}$ be the $\left(\sim_{\mathcal{U}}\right)$-equivalence class of $\left(x_{n}\right)$ and put

$$
\left(\prod_{n} X_{n}\right) / \mathcal{U}=\left\{\left[\left(x_{n}\right)\right]_{\mathcal{U}}:\left(x_{n}\right) \in \prod_{n} X_{n}\right\} .
$$

Given $\left(A_{n}\right) \in \prod_{n} \boldsymbol{B}\left(X_{n}\right)$, we define $\left[\left(A_{n}\right)\right]_{\mathcal{U}} \subseteq\left(\prod_{n} X_{n}\right) / \mathcal{U}$ by

$$
\left[\left(x_{n}\right)\right]_{\mathcal{U}} \in\left[\left(A_{n}\right)\right]_{\mathcal{U}} \Leftrightarrow \mathcal{U} n\left(x_{n} \in A_{n}\right) .
$$

Put

$$
\boldsymbol{B}_{\mathcal{U}}^{0}=\left\{\left[\left(A_{n}\right)\right]_{\mathcal{U}}:\left(A_{n}\right) \in \prod_{n} \boldsymbol{B}\left(X_{n}\right)\right\}
$$

Then $\boldsymbol{B}_{\mathcal{U}}^{0}$ is a Boolean algebra of subsets of $\left(\prod_{n} X_{n}\right) / \mathcal{U}$.

For $\left[\left(A_{n}\right)\right]_{\mathcal{U}} \in \boldsymbol{B}_{\mathcal{U}}^{0}$, put

$$
\mu_{\mathcal{U}}\left(\left[\left(A_{n}\right)\right]_{\mathcal{U}}\right)=\lim _{n \rightarrow \mathcal{U}} \mu_{n}\left(A_{n}\right),
$$

where $\lim _{n \rightarrow \mathcal{U}} y_{n}$ denotes the ultrafilter limit of the sequence $\left(y_{n}\right)$ of reals. Then $\mu_{\mathcal{U}}$ is a finitely additive probability Borel measure on $\boldsymbol{B}_{\mathcal{U}}^{0}$. This can be extended to a (countably additive) probability measure on a $\sigma$-algebra $\boldsymbol{B}_{\mathcal{U}}$ containing $\boldsymbol{B}_{\mathcal{U}}^{0}$, also denoted by $\mu_{\mathcal{U}}$. Then $\left(\left(\prod_{n} X_{n}\right) / \mathcal{U}, \mu_{\mathcal{U}}\right)$ is the ultraproduct of $\left(X_{n}, \mu_{n}\right)$. When $\left(X_{n}, \mu_{n}\right)$ are all equal to a fixed space $(X, \mu)$, we call this ultraproduct the ultrapower of $(X, \mu)$ and denote it by $X_{\mathcal{U}}$.

Let now $\Gamma$ be a countable group and let $a \in A(\Gamma, X, \mu)$. We can define then the action $\alpha_{\mathcal{U}}: \Gamma \times X_{\mathcal{U}} \rightarrow X_{\mathcal{U}}$ by

$$
\gamma^{a_{\mathcal{U}}} \cdot\left[\left(x_{n}\right)\right]_{\mathcal{U}}=\left[\left(\gamma^{a} \cdot x_{n}\right)\right]_{\mathcal{U}},
$$

where we let $\gamma^{a_{\mathcal{U}}} \cdot x=a_{\mathcal{U}}(\gamma, x)$ and similarly for $a$. This is a measure preserving action on the ultrapower $\left(X_{\mathcal{U}}, \mu_{\mathcal{U}}\right)$.

Consider now a sequence of measure preserving $\operatorname{CBER}\left(F_{n}\right)$ on $(X, \mu)$. Let $E \in \mathcal{E}$ be such that $F_{n} \subseteq E$, for each $n$. Fix an action $a \in A(\Gamma, X, \mu)$ such that $E_{a}=E$. We will use this to define an ultraproduct $\prod_{n}^{a} F_{n} / \mathcal{U}$ of the $F_{n}$. It turns out that it is independent of $E$ and the action $a$, so that we can define unambiguously the ultraproduct $\prod_{n} F_{n} / \mathcal{U}$.

We will use below the following general proposition:

Proposition 13.1. Let $\Gamma$ be a group, $a: \Gamma \times X \rightarrow X$ an action of $\Gamma$ on a set $X$ and put $a(\gamma, x)=\gamma \cdot x$. Let $E_{a}$ be the induced equivalence relation on $X$ and let $F \subseteq E_{a}$ be a subequivalence relation. For $\gamma \in \Gamma$, let

$$
A_{\gamma, F}^{a}=A_{\gamma, F}=\{x:(x, \gamma \cdot x) \in F\} .
$$

Then

(1) $A_{1, F}=X$,

(2) $A_{\gamma, F} \subseteq \gamma^{-1} \cdot A_{\gamma^{-1}, F}$, 
(3) $A_{\gamma, F} \cap \gamma^{-1} \cdot A_{\delta, F} \subseteq A_{\delta \gamma, F}$,

(4) $A_{\delta, F} \cap \operatorname{Fix}\left(\delta^{-1} \gamma\right) \subseteq A_{\gamma, F}$

where

$$
\operatorname{Fix}(p)=\{x: p \cdot x=x\} .
$$

Conversely, if $\left(A_{\gamma}\right)_{\gamma \in \Gamma}$ is a family of sets satisfying 1.-3. above, then the relation

$$
x F y \Longleftrightarrow \exists \gamma\left(\gamma \cdot x=y \vee x \in A_{\gamma}\right)
$$

defines a subequivalence relation of $E_{a}$ and if 4 . also holds we have that $A_{\gamma}=A_{\gamma, F}$.

Let now $A_{\gamma, F_{n}}^{a}=\left\{x \in X:\left(x, \gamma^{a}(x)\right) \in F_{n}\right\}$ and $A_{\gamma}^{a}=\left[\left(A_{\gamma, F_{n}}^{a}\right)\right]_{\mathcal{U}}$. Consider also the ultrapower $a_{\mathcal{U}}$. Then $\left(A_{\gamma}^{a}\right)$ satisfies conditions 1.-4. of Proposition 13.1 and therefore it gives rise to a countable equivalence relation $\hat{F}^{a}=\prod_{n}^{a} F_{n} / \mathcal{U}$ on $X_{\mathcal{U}}$ defined by

$$
\left[\left(x_{n}\right)\right]_{\mathcal{U}} \hat{F}^{a}\left[\left(y_{n}\right)\right]_{\mathcal{U}} \Longleftrightarrow \exists \gamma \in \Gamma\left(\gamma^{a_{\mathcal{U}}}\left(\left[\left(x_{n}\right)\right]_{\mathcal{U}}\right)=\left[\left(y_{n}\right)\right]_{\mathcal{U}} \&\left[\left(x_{n}\right)\right]_{\mathcal{U}} \in A_{\gamma}^{a}\right) .
$$

Thus $\prod_{n}^{a} F_{n} / \mathcal{U}$ is the union of the graphs of $\gamma^{a_{\mathcal{U}}} \mid A_{\gamma}^{a}, \gamma \in \Gamma$. It is easy to see that the equivalence relation induced by each $\gamma^{a_{\mathcal{U}}} \mid A_{\gamma}^{a}$ is also induced by a single measure preserving automorphism of $\left(X_{\mathcal{U}}, \mu_{\mathcal{U}}\right)$ and thus $\prod_{n}^{a} F_{n} / \mathcal{U}$ is induced by a measure preserving action of a countable group on $\left(X_{\mathcal{U}}, \mu_{\mathcal{U}}\right)$. Thus we can view $\prod_{n}^{a} F_{n} / \mathcal{U}$ as a countable, measure preserving equivalence relation on $\left(X_{\mathcal{U}}, \mu_{\mathcal{U}}\right)$. Note that we also have $A_{\gamma, \hat{F}^{a}}^{a_{\mathcal{U}}}=\left[\left(A_{\gamma, F_{n}}^{a}\right)\right]_{\mathcal{U}}$ and so $\mu_{\mathcal{U}}\left(A_{\gamma, \hat{F}^{a}}^{a_{\mathcal{U}}}\right)=\lim _{n \rightarrow \mathcal{U}} \mu\left(A_{\gamma, F_{n}}^{a}\right)$.

\section{Factors}

14.1. Factors in general. Let $E$ be a measure preserving CBER on $(X, \mu)$. Let $\mathcal{A} \subseteq \mathrm{MALG}_{\mu}$ be a non-atomic, $\sigma$-subalgebra of MALG. Put

$$
[E]^{\mathcal{A}}=\left\{T \in[E]: \forall A \in \mathcal{A}\left(T(A), T^{-1}(A) \in \mathcal{A}\right)\right\} .
$$

This is a closed subgroup of $([E], u)$, which we call the relative to $\mathcal{A}$ full group of $E$.

Consider now a separable subgroup $\Gamma$ of $(\operatorname{Aut}(X, \mu), u)$. This defines a measure preserving CBER $F^{\Gamma}$ as follows: Let $\Gamma_{0} \leq \Gamma$ be a countable dense subgroup of $\Gamma$ and let $F^{\Gamma}$ be the equivalence relation induced by $\Gamma_{0}$. We can easily see that this is independent of the choice of $\Gamma_{0}$ and moreover $\Gamma \leq\left[F^{\Gamma}\right]$.

Clearly $F^{\Gamma}$ is the smallest equivalence relation $F$ such that $\Gamma \leq[F]$. KittrellTsankov [KT, 4.14] have shown that if $\Gamma$ is also closed in the uniform topology, then there is a largest equivalence relation $F$, denoted by $F_{\Gamma}$, such that $[F] \leq \Gamma$ and moreover $\left[F_{\Gamma}\right]$ is a normal subgroup of $\Gamma$.

We now say that $E$ is generated by $\mathcal{A}$ or that $\mathcal{A}$ generates $E$ if $F^{[E]^{\mathcal{A}}}=E$ (clearly always $F^{[E]^{\mathcal{A}}} \subseteq E$ ). This is equivalent to saying that there is a countable group $\Gamma$ and an action $a \in A(\Gamma, X, \mu)$ such that $E_{a}=E$ and $\mathcal{A}$ is invariant under $a$, i.e., for each $A \in \mathcal{A}, \gamma \in \Gamma$ we have that $\gamma^{a}(A) \in \mathcal{A}$.

Assume $\mathcal{A}$ generates $E$ and let $\pi:(X, \mu) \rightarrow(Y, \nu)$ be the factor corresponding to $\mathcal{A}$, so that $(Y, \nu)$ is a standard (non-atomic) measure space, $\pi_{*} \mu=\nu$ and $B \mapsto$ $\pi^{-1}(B)$ is an isomorphism of $\left(\mathrm{MALG}_{\nu}, \nu\right)$ with $(\mathcal{A}, \mu \mid \mathcal{A})$ (see $\left.[\mathbf{K 2}, 17.43]\right)$. If $T \in$ $\operatorname{Aut}(X, \mu)$ preserves $\mathcal{A}$ (i.e., $\forall A \in \mathcal{A}\left(T(A), T^{-1}(A) \in \mathcal{A}\right)$ ), then (via $\pi^{-1}$ ) it gives 
an automorphism of $\mathrm{MALG}_{\nu}$, i.e., an element of $\operatorname{Aut}(Y, \nu)$, denoted by $\hat{\pi}(T)$, such that $\hat{\pi}(T)(\pi(x))=\pi(T(x))$. So if

$$
\operatorname{Aut}(X, \mu)^{\mathcal{A}}=\left\{T \in \operatorname{Aut}(X, \mu): \forall A \in \mathcal{A}\left(T(A), T^{-1}(A) \in \mathcal{A}\right)\right\},
$$

then $\operatorname{Aut}(X, \mu)^{\mathcal{A}}$ is a closed subgroup of $(\operatorname{Aut}(X, \mu), u)$ and

$$
\hat{\pi}:\left(\operatorname{Aut}(X, \mu)^{\mathcal{A}}, u\right) \rightarrow(\operatorname{Aut}(Y, \nu), u)
$$

is a continuous homomorphism. In particular, $\hat{\pi}\left([E]^{\mathcal{A}}\right)$ is a separable subgroup of (Aut $(Y, \nu), u)$ and thus gives rise to the equivalence relation $F=F^{\hat{\pi}\left([E]^{\mathcal{A}}\right)}$. We call this the factor of $E$ relative to $\mathcal{A}$.

Note that if $\Gamma_{0} \leq[E]^{\mathcal{A}}$ is dense in $\left([E]^{\mathcal{A}}, u\right)$, so that $\Gamma_{0}$ generates $E$, then $\hat{\pi}\left(\Gamma_{0}\right)$ is dense in $\left(\hat{\pi}\left([E]^{\mathcal{A}}\right), u\right)$ and so, by definition, it generates the factor $F$. It follows that there is a countable group $\Gamma$ and an action $a \in A(\Gamma, X, \mu)$ preserving $\mathcal{A}$ with $E_{a}=E$ such that if $\hat{\pi}(a)=b$ is the factor action of $a$ via $\pi$ (i.e., $\gamma^{b}=\hat{\pi}\left(\gamma^{a}\right)$ for each $\gamma \in \Gamma)$, so that

$$
\pi\left(\gamma^{a}(x)\right)=\gamma^{b}(\pi(x))
$$

then we have $E_{b}=F$. Therefore $\pi$ is a homomorphism of $E$ into $F$, i.e.,

$$
x E y \Rightarrow \pi(x) F \pi(y)
$$

and also $\pi$ is class-surjective, i.e., the image of each $E$-class is an $F$-class. Moreover if $c \in A(\Delta, X, \mu)$ is any action of a countable group $\Delta$ preserving $\mathcal{A}$ with $E_{c}=E$ and $\hat{\pi}(c)=d$ is the factor action of $c$ via $\pi$, then $E_{d}=F$.

Clearly $\hat{\pi}$ is a homomorphism of $[E]^{\mathcal{A}}$ into $[F]$. In fact we have:

Proposition 14.1. The homomorphism $\hat{\pi}:[E]^{\mathcal{A}} \rightarrow[F]$ is surjective.

The kernel of $\hat{\pi} \mid[E]^{\mathcal{A}}$ is equal to

$$
[E]_{\mathcal{A}}=\left\{T \in[E]^{\mathcal{A}}: \forall A \in \mathcal{A}(T(A)=A)\right\},
$$

thus $[F] \cong[E]^{\mathcal{A}} /[E]_{\mathcal{A}}$ (as topological groups). Note also that $T \in[E]_{\mathcal{A}} \Longleftrightarrow T \in$ $[E]^{\mathcal{A}} \wedge \pi(T(x))=\pi(x), \forall x$.

Let $R_{\pi}$ be the kernel of $\pi$, i.e., the smooth equivalence relation given by:

$$
x R_{\pi} y \Longleftrightarrow \pi(x)=\pi(y) \text {. }
$$

Put also

Thus $[E]_{\mathcal{A}}=\left[E_{\pi}\right]$.

$$
E_{\pi}=E \cap R_{\pi}
$$

It is easy to check that $E, R_{\pi}$ commute, i.e., $E \circ R_{\pi}=R_{\pi} \circ E$. (Here for any two equivalence relations $E_{1}, E_{2}$, we define the relation $E_{1} \circ E_{2}$ by $x E_{1} \circ E_{2} y \Longleftrightarrow$ $\exists z\left(x E_{1} z \wedge z E_{2} y\right)$.)

We now have:

Proposition 14.2. Let $F$ be a factor of $E$, let $S_{0}, S_{1}, \cdots \in[F]$ be such that $F=$ $E_{S_{0}, S_{1}, \ldots}$, and let $T_{0}, T_{1}, \cdots \in[E]^{\mathcal{A}}$ be such that $\hat{\pi}\left(T_{i}\right)=S_{i}$. If $E^{\prime}=E_{T_{0}, T_{1}, \ldots}$, then $E=E^{\prime} \vee E_{\pi}$.

The following result was shown by R. Tucker-Drob.

Proposition 14.3 (Tucker-Drob). Let $S \in[F]$ be an involution. Then there is an involution $T \in[E]^{\mathcal{A}}$ with $\hat{\pi}(T)=S$. 
Corollary 14.4. If $E$ is generated by the $\sigma$-subalgebra $\mathcal{A}$, then there are involutions $T_{0}, T_{1}, \cdots \in[E]^{\mathcal{A}}$ such that $E=E_{T_{0}, T_{1}, \ldots}$.

We next have the following.

Theorem 14.5. The composition of factors is a factor.

It also follows from the proof of Theorem 14.5 that there is a countable group $\Gamma$ and an action $a \in A(\Gamma, X, \mu)$, preserving both $\mathcal{A}$ and $\mathcal{C}$, such that $E_{a}=E$, and moreover if $\hat{\pi}(a)=b$, then $E_{b}=F$ and $b$ preserves $\mathcal{B}$ and if $\hat{\rho}(b)=\hat{\rho}(\hat{\pi}(a))=c$, then $E_{c}=H$.

This can be extended to infinite chains as follows.

For each $n \in \mathbb{N}$, let $E_{n}$ be an equivalence relation on $\left(X_{n}, \mu_{n}\right)$ and for each $n \geq 1$, let $\pi_{n}:\left(X_{n}, \mu_{n}\right) \rightarrow\left(X_{n-1}, \mu_{n-1}\right)$ be the map corresponding to a $\sigma$-subalgebra $\mathcal{A}_{n} \subseteq$ MALG $_{\mu_{n}}$, which generates $E_{n}$, and let $E_{n-1}$ be the factor corresponding to $\mathcal{A}_{n}$. For $n>m$, let $\pi_{n, m}=\pi_{m+1} \circ \cdots \circ \pi_{n-1} \circ \pi_{n}$ and let $\pi_{n, n}$ be the identity function on $X_{n}$. Then $\pi_{n, m}: X_{n} \rightarrow X_{m}$, for $n \geq m$. Put $\mathcal{A}_{n, m}=\pi_{n, m}^{-1}\left(\mathrm{MALG}_{\mu_{m}}\right)$, so that $\mathcal{A}_{n, n-1}=\mathcal{A}_{n}$ and $\mathcal{A}_{n, n}=$ MALG $_{\mu_{n}}$. Thus we have the following $\sigma$-subalgebras of $\mathrm{MALG}_{\mu_{n}}$

$$
\mathcal{A}_{n, 0} \subseteq \mathcal{A}_{n, 1} \subseteq \cdots \subseteq \mathcal{A}_{n, n-1}=\mathcal{A}_{n} \subseteq \mathcal{A}_{n, n}=\mathrm{MALG}_{\mu_{n}}
$$

Put

Then we have:

$$
\left[E_{n}\right]^{*}=\left[E_{n}\right]^{\mathcal{A}_{n, 0}} \cap \cdots \cap\left[E_{n}\right]^{\mathcal{A}_{n, n-1}}
$$

Proposition 14.6. For each $n \geq 1$,

(i) $\hat{\pi}_{n}\left(\left[E_{n}\right]^{*}\right)=\left[E_{n-1}\right]^{*}$,

(ii) $E_{n}=F^{\left[E_{n}\right]^{*}}$.

Consider now the inverse limit $\left(X_{\infty}, \mu_{\infty}\right)$ of the sequence $\left(X_{n}, \mu_{n}\right), \pi_{n}$. Denote by $\pi_{\infty, n}:\left(X_{\infty}, \mu_{\infty}\right) \rightarrow\left(X_{n}, \mu_{n}\right)$ the associated maps, so that $\pi_{n, m} \circ \pi_{\infty, n}=\pi_{\infty, m}$ for $n \geq m$. Thus $X_{\infty}$ consist of all chains $\left(x_{n}\right) \in \prod_{n} X_{n}$ with $\pi_{n}\left(x_{n}\right)=x_{n-1}$, for $n \geq 1, \pi_{\infty, n}\left(\left(x_{n}\right)\right)=x_{n}$ and $\mathrm{MALG}_{\mu_{\infty}}$ is the smallest $\sigma$-algebra containing the $\sigma$-subalgebras

$$
\mathcal{A}_{\infty, 0}=\pi_{\infty, 0}^{-1}\left(\mathrm{MALG}_{\mu_{0}}\right) \subseteq \mathcal{A}_{\infty, 1}=\pi_{\infty, 1}^{-1}\left(\mathrm{MALG}_{\mu_{1}}\right) \subseteq \ldots
$$

There is a countable group $\Gamma$ and a measure preserving action $a_{\infty} \in A\left(\Gamma, X_{\infty}, \mu_{\infty}\right)$, which keeps all the $\mathcal{A}_{\infty, n}$ invariant, thus factors to a measure preserving action $\hat{\pi}_{\infty, n}\left(a_{\infty}\right)=a_{n} \in A\left(\Gamma, X_{n}, \mu_{n}\right)$, which has moreover the property that $E_{a_{n}}=E_{n}$. Then if we put $E_{a_{\infty}}=E_{\infty}$, it follows that the factor of $E_{\infty}$ via $\pi_{\infty, n}$ is exactly $E_{n}$ and the appropriate diagrams commute.

Although $E_{\infty}$ is an "upper bound" for the inverse system $\left(E_{n}\right)$, it is not clear how to construct a canonical upper bound, i.e., an inverse limit in the categorical sense for this inverse system.

Next we see that hyperfiniteness is preserved under factoring.

Proposition 14.7. If $E$ is hyperfinite and $F$ is a factor of $E$, then $F$ is hyperfinite.

This result can be used, along with an ultraproduct argument, to give a different proof of a strengthening concerning weak containment of actions, due to R. TuckerDrob (private communication). We first need a proposition which extends Proposition 5.7 of $[\mathbf{C K T}]$ and Corollary 3.1 of $[\mathbf{A E}]$. Below we let $a \simeq b \Longleftrightarrow a \preceq b \& b \preceq a$ 
denote the weak equivalence of the actions $a, b$ and $a \sqsubseteq b$ denote that the action $a$ is a factor of the action $b$.

Proposition 14.8. Let $\Gamma, \Delta$ be infinite countable groups, $a, b \in A(\Gamma, X, \mu), c \in$ $A(\Delta, X, \mu)$ be such that $a \preceq b$ and $E_{b} \subseteq E_{c}$. Then there are $d \in A(\Gamma, X, \mu)$, $e \in A(\Delta, X, \mu)$ such that $b \simeq d, c \simeq e, a \sqsubseteq d$ and $E_{d} \subseteq E_{e}$. Similarly replacing $E_{b} \subseteq E_{c}, E_{d} \subseteq E_{e}$ by $E_{b}=E_{c}, E_{d}=E_{e}$, resp.

Taking now $\Delta=\mathbb{Z}$, this gives the following:

Theorem 14.9 (Tucker-Drob). Let $\Gamma$ be an infinite countable group and $a, b \in$ $A(\Gamma, X, \mu)$ be such that $a \preceq b$. If $E_{b}$ is hyperfinite, then $E_{a}$ is hyperfinite.

14.2. Class-bijective factors. We now consider the following notion that has been considered in the literature, see Feldman-Sutherland-Zimmer [FSZ]). A measure preserving CBER $F$ on $(Y, \nu)$ is called a class-bijective factor of a measure preserving CBER $E$ on $(X, \mu)$ if there is Borel $\pi:(X, \mu) \rightarrow(Y, \nu)$ with $\pi_{*} \mu=\nu$, $\pi: E \rightarrow F$ a homomorphism (i.e., $x E x^{\prime} \Rightarrow \pi(x) F \pi\left(x^{\prime}\right)$ ) such that moreover for each $E$-class $[x]_{E}$ the map $\pi$ is a bijection of $[x]_{E}$ with $[\pi(x)]_{F}$. In this case we also call the map $\pi$ class-bijective. For example, let $E$ be measure preserving on $(X, \mu), \mathcal{A} \subseteq$ MALG $_{\mu}$ a $\sigma$-subalgebra which generates $E, \pi:(X, \mu) \rightarrow(Y, \nu)$ the corresponding map, $a \in A(\Gamma, X, \mu)$ with $E_{a}=E$ leaving $\mathcal{A}$ invariant, $\hat{\pi}(a)=b$ and $F=E_{b}$. If $b$ is free, then $F$ is a class-bijective factor of $E$.

Proposition 14.10. A class-bijective factor is a factor in the sense of Section 14.1.

Thus a class-bijective factor is a factor $\pi$ for which $E_{\pi}=i d$, where $i d$ is the equality relation. In fact it turns out that the class-bijective factors of a measure preserving CBER $E$ on $(X, \mu)$ correspond exactly to smooth equivalence relations $R_{\pi}$ that commute with $E$ and are orthogonal to $E$ in the sense that $E_{\pi}=R_{\pi} \cap E=$ $i d$.

Since $\left[E_{\pi}\right]=[E]_{\mathcal{A}}$ we also immediately have:

Proposition 14.11. Assume that $E$ on $(X, \mu)$ is generated by the $\sigma$-subalgebra $\mathcal{A} \subseteq$ MALG $_{\mu}$ with corresponding map $\pi:(X, \mu) \rightarrow(Y, \nu)$ and factor $F$. Then $\pi$ is class-bijective iff $[E]_{\mathcal{A}}$ is trivial, i.e., $\hat{\pi}$ is an isomorphism of $[E]^{\mathcal{A}}$ with $[F]$.

We will next characterize which factors are class-bijective. Below for each $T \in$ $\operatorname{Aut}(X, \mu)$, we let as usual $\operatorname{supp}(T)=\{x: T(x) \neq x\}$.

Proposition 14.12. Assume that $E$ on $(X, \mu)$ is generated by the $\sigma$-subalgebra $\mathcal{A} \subseteq \mathrm{MALG}_{\mu}$, with corresponding map $\pi:(X, \mu) \rightarrow(Y, \nu)$ and factor $F$. Then $\pi$ is class-bijective iff for each $T \in[E]^{\mathcal{A}}, \operatorname{supp}(T)=\pi^{-1}(\operatorname{supp}(\hat{\pi}(T)))$.

From Proposition 14.12, and using its notation, we see that if $\pi$ is class-bijective, then for $T \in[E]^{\mathcal{A}}$ we have that $\operatorname{supp}(T) \in \mathcal{A}$. Conversely this last condition almost characterizes class-bijective factors. Recall that $\pi$ is class-bijective iff $\operatorname{card}\left([x]_{E_{\pi}}\right)=$ 1 , for all $x$.

Proposition 14.13. Assume that $E$ on $(X, \mu)$ is generated by the $\sigma$-subalgebra $\mathcal{A} \subseteq \mathrm{MALG}_{\mu}$, with corresponding factor map $\pi:(X, \mu) \rightarrow(Y, \nu)$. If for every $T \in[E]^{\mathcal{A}}, \operatorname{supp}(T) \in \mathcal{A}$, then $\operatorname{card}\left([x]_{E_{\pi}}\right) \leq 2$, for all $x$. 
The conclusion of Proposition 14.13 cannot be strengthened to $\pi$ being classbijective.

Class-bijective factors can be also characterized, in the ergodic case, in terms of skew products. Let $F$ be a measure preserving $\operatorname{CBER}$ on $(Y, \nu)$. Let $(Z, \sigma)$ be a standard, not necessarily non-atomic, measure space and let $\alpha: F \rightarrow \operatorname{Aut}(Z, \sigma)$ be a Borel cocycle, i.e., $\alpha(x, z)=\alpha(y, z) \alpha(x, y)$ for $x F y F z$ (in an $F$-invariant set of measure 1). Let $X=Y \times Z, \mu=\nu \times \sigma$ and define the skew product equivalence relation $E$ on $X$, in symbols

$$
E=F \times_{\alpha}(Z, \sigma)
$$

by

$$
(x, z) E(y, w) \Longleftrightarrow x F y \& \alpha(x, y)(z)=w .
$$

Let $p: X \rightarrow Y$ be the projection map $p(y, z)=y$. Let $a \in A(\Gamma, Y, \nu)$ be such that $E_{a}=F$. Let also $\alpha^{*}(g, y)=\alpha\left(y, g^{a}(y)\right)$. Then if $b=a \times_{\alpha^{*}}(Z, \sigma)$ is the skew product action (see $[\mathbf{K}$, Section $10,(\mathbf{E})]$ ), we have $E_{b}=E$ and since $\hat{p}(b)=a$, it follows that $F$ is the factor of $E$ corresponding to $p$. Moreover it is easy to see that it is class-bijective.

Conversely, the proof of Rokhlin's Skew Product Theorem (see Glasner [Gl], 3.18 ) shows that if $F$ on $(Y, \nu)$ is a class-bijective factor of an ergodic $E$ on a space $(X, \mu)$ via $\pi:(X, \mu) \rightarrow(Y, \nu)$, then there is a standard, not necessarily non-atomic, space $(Z, \sigma)$, a Borel cocycle $\alpha: F \rightarrow \operatorname{Aut}(Z, \sigma)$ and an isomorphism $\varphi:(X, \mu) \rightarrow$ $(Y \times Z, \nu \times \sigma)$ of $E$ with $F \times_{\alpha}(Z, \sigma)$ such that $p \circ \varphi=\pi$.

If $F$ on $(Y, \nu)$ is a (class-bijective) factor of $E$ on $(X, \mu)$ via $\pi$, we say that $E$ is a (class-bijective) extension of $E$ via $\pi$. Given two such extensions $E, E^{\prime}$ of $F$ on $(X, \mu),\left(X^{\prime}, \mu^{\prime}\right)$ via $\pi, \pi^{\prime}$, we say that they are isomorphic if there is an isomorphism $\varphi:(X, \mu) \rightarrow\left(X^{\prime}, \mu^{\prime}\right)$ of $E$ with $E^{\prime}$ with $\pi^{\prime} \circ \varphi=\pi$. Thus we see the following:

Theorem 14.14. Let $F$ be an ergodic measure preserving CBER on $(Y, \nu)$. Let $E$ be an ergodic extension of $F$ on $(X, \mu)$ via $\pi:(X, \mu) \rightarrow(Y, \nu)$. Then the following are equivalent:

(i) $E, \pi$ is a class-bijective extension of $F$.

(ii) $E, \pi$ is isomorphic to a skew product extension of $F$.

Concerning the question of inverse limits for systems $\left(\left(X_{n}, \mu_{n}\right), \pi_{n}, E_{n}\right)$, we note that if we restrict ourselves to the category of class-bijective factors, i.e., if in this system every factor is class-bijective, then it is easy to see that there is indeed a canonical inverse limit $E_{\infty}=\varliminf_{n} E_{n}$ on $\left(X_{\infty}, \mu_{\infty}\right)$, given by

$$
\left(x_{n}\right) E_{\infty}\left(y_{n}\right) \Longleftrightarrow \forall n\left(x_{n} E_{n} y_{n}\right) \text {. }
$$

This follows from the "unique lifting property" given in Proposition 14.11, which implies that if $a_{0} \in A\left(\Gamma, X_{0}, \mu_{0}\right)$ is such that $E_{a_{0}}=E_{0}$, then there are unique $a_{n} \in A\left(\Gamma, X_{n}, \mu_{n}\right)$ with $\hat{\pi}_{n, m}\left(a_{n}\right)=a_{m}$ for $n \geq m$ and $a_{\infty} \in A\left(\Gamma, X_{\infty}, \mu_{\infty}\right)$ with $\hat{\pi}_{\infty, n}\left(a_{\infty}\right)=a_{n}$ such that $E_{a_{n}}=E_{n}, E_{a_{\infty}}=E_{\infty}$.

The following is an interesting open problem. Recall that a measure preserving CBER is treeable if it admits an acyclic Borel graphing.

Problem 14.15. If $E$ is treeable and $F$ is a class-bijective factor of $E$, is $F$ treeable? 
Note that a positive answer implies that every countable treeable group $\Gamma$ is strongly treeable. (Recall that a countable group $\Gamma$ is treeable if there is some free $a \in A(\Gamma, X, \mu)$ with $E_{a}$ treeable, while it is strongly treeable if this holds for every free $a \in A(\Gamma, X, \mu)$.) Indeed let $a \in A(\Gamma, X, \mu)$ be free with $E_{a}$ treeable and consider any free $b \in A(\Gamma, Y, \nu)$. Let $a \times b$ be the product of $a, b$. Then $E_{a \times b}$ is a class-bijective extension of $E_{a}$, so it is treeable. Also $E_{b}$ is a class-bijective factor of $E_{a \times b}$, so, if the answer to Problem 14.15 is positive, $E_{b}$ is treeable.

14.3. Other notions of factors. In the preceding we have considered two categories whose objects are triples $(X, \mu, E)$, with $E$ a countable measure preserving Borel equivalence relation on $(X, \mu)$.

(1) In the first category, the morphisms $\pi:(X, \mu, E) \rightarrow(Y, \nu, F)$ are measure preserving Borel maps $\pi:(X, \mu) \rightarrow(Y, \nu)$ with $\pi: E \rightarrow F$ a class-bijective homomorphism, i.e., for each $x \in X, \pi$ is a bijection of $[x]_{E}$ with $[\pi(x)]_{E}$. (The notation $\pi: E \rightarrow F$, which more accurately should be written as $\pi \times \pi: E \rightarrow F$, indicates that $\pi$ is a homomorphism of $E$ into $F$.)

(2) In the second category, the morphisms $\pi:(X, \mu, E) \rightarrow(Y, \nu, F)$ are measure preserving Borel maps $\pi:(X, \mu) \rightarrow(Y, \nu)$ such that if $\mathcal{A} \subseteq$ MALG $_{\mu}$ is the $\sigma$-algebra associated to $\pi$, then $[E]^{\mathcal{A}}$ generates $E$ and $\hat{\pi}\left([E]^{\mathcal{A}}\right)$ generates $F$ (or equivalently there is Borel action $a$ of a countable group $\Gamma$ preserving $\mathcal{A}$, such that $E_{a}=E$ and $\left.E_{\hat{\pi}(a)}=F\right)$.

R. Tucker-Drob (unpublished) considered the following two additional categories with the same objects $(X, \mu, E)$.

(3) In the third category, the morphisms $\pi:(X, \mu, E) \rightarrow(Y, \nu, F)$ are measure preserving Borel maps $\pi:(X, \mu) \rightarrow(Y, \nu)$ with $\pi: E \rightarrow F$ a class-surjective homomorphism, i.e., for each $x \in X, \pi$ is a surjection of $[x]_{E}$ with $[\pi(x)]_{E}$. A class-surjective homomorphism is a morphism in the sense of the second category (i.e., that of Section 14.1) iff the homomorphism $\hat{\pi}:[E]^{\mathcal{A}} \rightarrow[F]$ is surjective.

(4) Finally, in the fourth category, the morphisms $\pi:(X, \mu, E) \rightarrow(Y, \nu, F)$ are measure preserving Borel maps $\pi:(X, \mu) \rightarrow(Y, \nu)$ with $\pi: E \rightarrow F$ a surjective homomorphism (i.e., $(\pi \times \pi)(E)=F)$.

Note that the categories above have the same objects but increasingly more general morphisms.

14.4. An application to soficity. We start with the following proposition.

Proposition 14.16. Let $\pi: E \rightarrow F$ be a class-surjective homomorphism. Assume that $F$ is treeable. Then the following are equivalent:

(i) $F$ is a factor of $E$ via $\pi$.

(ii) There is Borel $E^{\prime} \subseteq E$ such that $F$ is a class-bijective factor of $E^{\prime}$ via $\pi$ and $E=E^{\prime} \vee E_{\pi}$.

Let $F$ be a measure preserving CBER on $(Y, \nu)$. We say that $F$ is unfoldable if for any $E$ on $(X, \mu)$ which factors to $F$ via $\pi:(X, \mu) \rightarrow(Y, \nu)$, there is $E^{\prime} \subseteq E$ such that $F$ is a class-bijective factor of $E^{\prime}$ via $\pi$. 
Thus every treeable equivalence relation is unfoldable. For the next result recall the notion of a sofic equivalence relation introduced in $[\mathbf{E L}]$. See also $[\mathbf{C K T}$, Definition 10.1] for an alternative description due to Ozawa.

Proposition 14.17. Every unfoldable equivalence relation is sofic.

The combination of Proposition 14.16 and Proposition 14.17 gives then a new proof of the following result of Elek-Lippner (another proof is also given in $[\mathbf{C K T}$, Section 10.3]).

Theorem 14.18 (Elek-Lippner, $[\mathbf{E L}]$ ). Every treeable equivalence relation is sofic.

14.5. Relative hyperfiniteness. We consider here the following question:

Suppose $E$ is hyperfinite and generated by a non-atomic $\sigma$-subalgebra $\mathcal{A}$, i.e., $E$ is generated by a countable group of transformations that are $\mathcal{A}$-measurable (i.e., preserve $\mathcal{A}$ ). Can we find a single $\mathcal{A}$-measurable transformation that generates $E$, i.e., is $E$ hyperfinite relative to $\mathcal{A}$ ?

The answer is in general negative. The following result provides the next possible answer.

Theorem 14.19. Let $E$ be hyperfinite and generated by a non-atomic $\sigma$-subalgebra A. Then

(i) There are $T_{1}, T_{2} \in[E]^{\mathcal{A}}$ that generate $E$.

(ii) If $E$ is ergodic, then there is $T \in[E]^{\mathcal{A}}$ that generates $E$ iff the factor corresponding to $\mathcal{A}$ is class-bijective.

Ben Miller raised the following questions:

Problem 14.20. i) Let $E$ be hyperfinite and generated by a non-atomic $\sigma$-subalgebra $\mathcal{A}$. Is there is an increasing sequence $E_{0} \subseteq E_{1} \subseteq \ldots$ of finite equivalence relations which are generated by $\mathcal{A}$ with $E=\bigcup_{n} E_{n}$ ?

ii) What if we assume the stronger hypothesis that $E=E_{T}$, for some $T \in[E]^{\mathcal{A}}$ ?

We have the following result which provides a weaker version of a positive answer to part i) of Problem 14.20 and a positive answer to part ii)..

Proposition 14.21. i) Let $E$ be hyperfinite and generated by a non-atomic $\sigma$ subalgebra $\mathcal{A}$. Then there is an increasing sequence $E_{0} \subseteq E_{1} \subseteq \ldots$ of equivalence relations, which are generated by $\mathcal{A}$, with $E=\bigcup_{n} E_{n}$ and for each $n$ an increasing sequence $E_{n, 0} \subseteq E_{n, 1} \subseteq \ldots$ of finite equivalence Borel relations which are generated by $\mathcal{A}$ such that $E_{n}=\bigcup_{m} E_{n, m}$.

In particular, $E$ is the limit (in the topology of $S(E)$ ) of a sequence of finite subequivalence relations which are generated by $\mathcal{A}$.

ii) If moreover $E=E_{T}$, for some $T \in[E]^{\mathcal{A}}$, then then there is an increasing sequence $E_{0} \subseteq E_{1} \subseteq \ldots$ of finite Borel equivalence relations, which are generated by $\mathcal{A}$, with $E=\bigcup_{n} E_{n}$.

One can also ask if a kind of converse of the Problem 14.20, ii) is true: If there is an increasing sequence $E_{0} \subseteq E_{1} \subseteq \ldots$ of finite Borel equivalence relations, which are generated by $\mathcal{A}$, with $E=\bigcup_{n} E_{n}$, is there $T \in[E]^{\mathcal{A}}$ such that $E=E_{T}$ ? This fails in general. 
Remark 14.22. Let $E$ be a measure preserving CBER on $(X, \mu)$. Then of course the following are equivalent:

a) $E=E_{T}$ for some $T \in \operatorname{Aut}(X, \mu)$;

b) $E$ is the union of an increasing sequence of finite Borel equivalence relations.

The preceding show that when relativized to a $\sigma$-subalgebra $\mathcal{A}$, a) implies b) but not vice versa.

14.6. Relative cost. Let $E$ be a measure preserving CBER on $(X, \mu)$ and let $\mathcal{A}$ be a non-atomic $\sigma$-subalgebra of $\mathrm{MALG}_{\mu}$ such that $E$ is generated by $\mathcal{A}$. Let $\pi: X \rightarrow Y$ be the associated to $\mathcal{A}$ factor map and $F$ the factor equivalence relation. Define the relative to $\mathcal{A}$ full pseudogroup of $E$, in symbols $[[E]]^{\mathcal{A}}$, as the set of all partial Borel bijections $\theta \in[[E]], \theta: A \rightarrow B$, such that $A, B \in \mathcal{A}$, and for any $A^{\prime} \subseteq A, B^{\prime} \subseteq B, A^{\prime}, B^{\prime} \in \mathcal{A}$, we have $\theta\left(A^{\prime}\right), \theta^{-1}\left(B^{\prime}\right) \in \mathcal{A}$. If $\theta \in[[E]]^{\mathcal{A}}, \theta: A \rightarrow B$, and $A=\pi^{-1}(C), B=\pi^{-1}(D)$, then, as in Section 14.1, we have an element $\hat{\pi}(\theta) \in[[F]]$ such that $\hat{\pi}(\theta): C \rightarrow D$ and $\hat{\pi}(\theta)(\pi(x))=\pi(\theta(x))$, for $x \in A$. Moreover the map $\hat{\pi}:[[E]]^{\mathcal{A}} \rightarrow[[F]]$ is surjective and preserves composition.

Next define the cost of $E$ relative to $\mathcal{A}$ by

$$
C^{\mathcal{A}}(E)=\inf \left\{\sum_{i \in I} \mu\left(A_{i}\right): \theta_{i}: A_{i} \rightarrow B_{i} \in[[E]]^{\mathcal{A}},\left(\theta_{i}\right)_{i \in I} \text { generates } E\right\}
$$

(where $I$ varies over countable index sets).

Clearly $C^{\mathcal{A}}(E) \geq C(E)$. Also notice that if $\left(\theta_{i}\right)_{i \in I}$ generates $E$, then $\left(\hat{\pi}\left(\theta_{i}\right)\right)_{i \in I}$ generates $F$, therefore $C^{\mathcal{A}}(E) \geq C(F)$.

Below we say that an equivalence relation $E$ on $(X, \mu)$ is finitely generated if it is of the form $E=E_{T_{1}, \ldots, T_{n}}$, for some $T_{1}, \ldots, T_{n} \in \operatorname{Aut}(X, \mu)$.

Theorem 14.23. Let $E$ be a measure preserving CBER on $(X, \mu)$ and let $\mathcal{A}$ be a non-atomic $\sigma$-subalgebra of MALG that generates $E$. Let $\pi:(X, \mu) \rightarrow(Y, \nu)$ be the associated to $\mathcal{A}$ factor map and $F$ the factor equivalence relation. If $F$ is aperiodic (e.g., if $E$ is ergodic) and $E_{\pi}$ is finitely generated, then $C^{\mathcal{A}}(E)=C(F)$.

Corollary 14.24. Let $E, \mathcal{A}, \pi, F$ be as in Theorem 14.23. Then if $E_{\pi}$ is hyperfinite, $C^{\mathcal{A}}(E)=C(F)$. In particular, if $E$ is ergodic hyperfinite, then $C^{\mathcal{A}}(E)=1$.

Although for $E$ ergodic hyperfinite there might not be a single automorphism $T \in[E]^{\mathcal{A}}$ that generates $E$ (see Theorem 14.19), Corollary 14.24 shows that $C^{\mathcal{A}}(E)$ is still equal to 1 .

It turns out that the hypothesis that $E_{\pi}$ is finitely generated is needed in Theorem 14.23.

We next consider the question of when the infimum in the definition of $C^{\mathcal{A}}(E)$ is attained.

Proposition 14.25. Let $E, \mathcal{A}, \pi, F$ be as in Theorem 14.23. Then

i) If $\pi$ is class-bijective and $F$ is treeable, the infimum in the definition of $C^{\mathcal{A}}(E)$ is attained.

ii) Conversely, if the infimum in the definition of $C^{\mathcal{A}}(E)$ is attained and $F$ has finite cost, $F$ is treeable and $\pi$ is class-bijective.

In particular, using also Proposition 14.7, if $E$ is ergodic hyperfinite, then the infimum in the definition of $C^{\mathcal{A}}(E)=1$ is attained iff $\pi$ is class-bijective. 
Let $E$ be a measure preserving CBER. We define the cost spectrum of $E$, in symbols $C S p(E)$, as the set of all $C^{\mathcal{A}}(E)$, where $\mathcal{A}$ varies over all the nonatomic $\sigma$-subalgebras of $\mathrm{MALG}_{\mu}$ such that $E$ is generated by $\mathcal{A}$. (Thus $C S p(E) \subseteq$ $[C(E), \infty]$.) Clearly the cost spectrum is an invariant of isomorphism among equivalence relations. It might therefore be interesting to study its structure.

For example, if $E$ is ergodic hyperfinite, then $\operatorname{CSp}(E)=\{1\}$. Is it true that if $E$ is ergodic, non-hyperfinite but has cost 1, then $\operatorname{CSp}(E) \neq\{1\}$ ? If in fact for every ergodic, non-hyperfinite $E$ of $\operatorname{cost} 1$, one has an $\mathcal{A}, \pi$ such that actually $E_{\pi}$ is finitely generated and $C^{\mathcal{A}}(E)>1$, then it follows that for every ergodic, non-hyperfinite $E$ there is a subequivalence relation induced by a free action of $\mathbb{F}_{2}$ (which answers positively [KM, 28.14]). Indeed if that is the case, every ergodic, non-hyperfinite $E$ would have a factor $F$ of cost $>1$, so that by [KM, 28.8] it would have a subequivalence relation induced by a free action of $\mathbb{F}_{2}$, which then could be lifted to such an action of $\mathbb{F}_{2}$ whose corresponding equivalence relation is included in $E$.

It is actually easy, using Theorem 14.23, to construct examples of ergodic, nonhyperfinite $E$ of cost 1, whose cost spectrum contains any finite set of reals $>1$.

14.7. Topological rank of relative full groups. Recall that a topological generator of a topological group $\Gamma$ is a subset $\Gamma_{0}$ of $\Gamma$ such that the subgroup generated by $\Gamma_{0}$ is dense in $\Gamma$. The topological rank of $\Gamma$, denoted by $t(\Gamma)$, is the smallest cardinality of a topological generator of $\Gamma$. Thus if $\Gamma$ is Polish, then $t(\Gamma) \leq \aleph_{0}$. It is easy to see that if $\Gamma$ is a Polish group, $N \triangleleft \Gamma$ a closed normal subgroup and $H=\Gamma / N$, then $t(\Gamma) \leq t(N)+t(H)$. Indeed, if $N_{0}$ is a topological generator of $N$ and $H_{0}$ a topological generator of $H$, then choose for each coset in $H_{0}$ a representative and let $\hat{H}_{0} \subseteq \Gamma$ consist of these representatives. Then $N_{0} \cup \hat{H}_{0}$ is a topological generator for $\Gamma$.

Let now $E$ be a measure preserving $\operatorname{CBER}$ on $(X, \mu)$, let $\mathcal{A}$ be a non-atomic $\sigma$-subalgebra of MALG, with associated map $\pi$, such that $E$ is generated by $\mathcal{A}$, and let $F$ be the factor of $E$ determined by $\mathcal{A}$. Then we have that

$$
t([F]) \leq t\left([E]^{\mathcal{A}}\right) \leq t([F])+t\left(\left[E_{\pi}\right]\right) .
$$

If then $F, E_{\pi}$ are aperiodic, we have $t([F]), t\left(\left[E_{\pi}\right]\right)=2$ (see [LeM, p. 263]), so $t\left([E]^{\mathcal{A}}\right) \leq 4$. We do not know if 4 here can be lowered to 2 .

\section{The Space of Graphs}

Consider again a measure preserving CBER $E$ on $(X, \mu)$. Denote by $\operatorname{Gr}(E)$ the set of all (simple, undirected) Borel graphs $G$ on $X$ such that $G \subseteq E$, where again we identify two such graphs if they agree a.e.

We will define again a canonical topology on $\operatorname{Gr}(E)$, which we call the topology of $\operatorname{Gr}(E)$, which, as in the case of equivalence relations, has several equivalent descriptions. (Thanks to A. Tserunyan for pointing out the equivalence of (1) and (2) below.)

(1) (The strong topology) For any $G \in G r(E)$ and $T \in[E]$, let again

$$
A_{T, G}=\{x:(x, T(x)) \in G\}
$$


and define the strong topology on $G r(E)$ as the one generated by the maps

$$
\begin{aligned}
G & \mapsto A_{T, G}, \\
G r(E) & \rightarrow \text { MALG }_{\mu},
\end{aligned}
$$

for $T \in[E]$.

This topology is again Polish. For this topology we have the following:

$$
\begin{aligned}
G_{n} \rightarrow G & \Longleftrightarrow \forall i\left(A_{T_{i}, G_{n}} \stackrel{\mathrm{MALG}_{\mu}}{\longrightarrow} A_{T_{i}, G}\right) \\
& \Longleftrightarrow \forall T \in[E]\left(A_{T, G_{n}} \stackrel{\mathrm{MALG}_{\mu}}{\longrightarrow} A_{T, G}\right) \\
& \Longleftrightarrow \forall \varphi \in[[E]]\left(A_{\varphi, G_{n}} \stackrel{\mathrm{MALG}_{\mu}}{\longrightarrow} A_{\varphi, G}\right),
\end{aligned}
$$

where, as usual, $\left(T_{i}\right)_{i \in \mathbb{N}}$ generates $E$ and for $\varphi \in[[E]], A_{\varphi, G}=\{x \in \operatorname{dom}(\varphi):(x, \varphi(x)) \in$ $G\}$.

(2) (The weak topology) One can also define the weak topology on $G r(E)$ as the topology generated by the maps $G \mapsto \mu\left(A_{T, G}\right), G r(E) \rightarrow[0,1]$, for $T \in[E]$.

(3) (A topology from the measure algebra of $E$ ) We can also view $G r(E)$ as a closed subspace of $\mathrm{MALG}_{E}$ with the induced topology.

Note again that if $G_{0} \subseteq G_{1} \subseteq \ldots, G=\bigcup_{n} G_{n}$, then $G_{n} \rightarrow G$ and similarly if $G_{0} \supseteq G_{1} \ldots, G=\bigcap_{n} G_{n}$.

Also the uniform topology on $\operatorname{Gr}(E)$ can be defined as in Section 2.7.

Remark 15.1. On the set of bounded degree graphs in $\operatorname{Gr}(E)$ one can also define the metric

$$
D(G, H)=M(G \triangle H)=\int|G(x) \triangle H(x)| d \mu(x)
$$

(see Lovász [L, page 352]), where $M$ is the measure on $E$ and $G(x)=\{y:(x, y) \in$ $G\}$ is the set of neighbors of $x$ in $G$. This gives rise to another topology on this set of graphs, for which is easy to check that it is at least as strong as the relative topology inherited from $\operatorname{Gr}(E)$ (i.e., contains the relative topology). However, even for graphs of degree at most 2 , it is easy to see that it may be actually strictly stronger.

However if we consider the set of all $d$-regular graphs, for fixed $d \geq 2$, the $D$ topology on that set agrees with its relative topology from $\operatorname{Gr}(E)$.

We also have the following analog of Theorem 2.9.

Theorem 15.2. Let $G_{n}, G \in G r(E)$ and $G_{n} \rightarrow G$. Then for each $i$, there is an increasing sequence $n_{0}^{(i)}<n_{1}^{(i)}<\ldots$, so that $\left(n_{m}^{(i+1)}\right)_{m \in \mathbb{N}}$ is a subsequence of $\left(n_{m}^{(i)}\right)_{m \in \mathbb{N}}$ and

$$
G=\bigcup \bigcap_{m \geq m} G_{n_{k}^{(m)}} .
$$

For $\mathcal{G} \subseteq \operatorname{Gr}(E)$ we define $\mathcal{G}_{\uparrow}, \mathcal{G}_{\downarrow}$ as in the case of equivalence relations. Then we have:

Theorem 15.3. If $\mathcal{G} \subseteq G r(E)$ is closed under finite intersections, then $\overline{\mathcal{G}}=\left(\mathcal{G}_{\downarrow}\right)_{\uparrow}$. 
A locally countable Borel graph $G$ on $X$ is $(\mu-)$ measure preserving if any partial Borel isomorphism $\varphi: A \rightarrow B$ such that $\operatorname{graph}(\varphi) \subseteq G$ is measure preserving. This is equivalent to saying that the equivalence relation generated by $G$ (i.e., the equivalence relation whose equivalence classes are the connected components of $G$ ) is measure preserving. Denote by $\mathcal{G} \mathcal{R}$ the set of all Borel locally countable, measure preserving graphs on $(X, \mu)$, where as usual we identify two such graphs if they agree a.e. Then $\operatorname{Gr}(E)=\{G \in \mathcal{G R}: G \subseteq E\}$ and $\mathcal{G R}=\bigcup_{E \in \mathcal{E}} G r(E)$. As in Section 3, we can see that if $E \subseteq F$, then $G r(E)$ is a closed subset of $G r(F)$ and the topology of $\operatorname{Gr}(E)$ is the relative topology it inherits form $\operatorname{Gr}(F)$. Thus, as in Section 3 , we can define the weak topology on $\mathcal{G R}$.

As a final comment, we mention that ultraproducts of graphs can be defined as in Section 13 using the following proposition:

Proposition 15.4. Let $\Gamma$ be a group, $a: \Gamma \times X \rightarrow X$ an action of $\Gamma$ on a set $X$ and put $a(\gamma, x)=\gamma \cdot x$. Let $E_{a}$ be the induced equivalence relation on $X$ and let $G \subseteq E_{a}$ be a graph. For $\gamma \in \Gamma$, let

$$
A_{\gamma, G}^{a}=A_{\gamma, G}=\{x:(x, \gamma \cdot x) \in G\} .
$$

Then

(1) $A_{1, G}=\emptyset$,

(2) $A_{\gamma, G} \subseteq \gamma^{-1} \cdot A_{\gamma^{-1}, G}$,

(3) $A_{\delta, G} \cap \operatorname{Fix}\left(\delta^{-1} \gamma\right) \subseteq A_{\gamma, G}$,

where

$$
\operatorname{Fix}(p)=\{x: p \cdot x=x\} .
$$

Conversely, if $\left(A_{\gamma}\right)_{\gamma \in \Gamma}$ is a family of sets satisfying 1.-2. above, then the relation

$$
x G y \Longleftrightarrow \exists \gamma\left(\gamma \cdot x=y \vee x \in A_{\gamma}\right)
$$

defines a graph contained in $E_{a}$ and if 3. also holds we have that $A_{\gamma}=A_{\gamma, G}$.

\section{More Complexity Calculations}

For each $G \in G r(E)$, let $G^{*} \in S(E)$ be the equivalence relation generated by $G$. The operation $G \in G r(E) \mapsto G^{*} \in S(E)$ is in general not continuous but we have the following:

Proposition 16.1. The map $\operatorname{Gr}(E) \ni G \mapsto G^{*} \in S(E)$ is of Baire class 1.

Recall that we call $G \in G r(E)$ a graphing of $E$ is $G^{*}=E$.

Theorem 16.2. The set $\{G \in G r(E): G$ is a graphing of $E\}$ is $G_{\delta}$ in $G r(E)$. If $E$ is aperiodic, it is also dense in $\operatorname{Gr}(E)$.

As in Section 7 , if $\mathcal{G} \subseteq \mathcal{G R}$ is a class of measure preserving locally countable Borel graphs and $E \in \mathcal{E}$, we let

$$
\mathcal{G}_{E}=\mathcal{G} \cap \operatorname{Gr}(E) .
$$

In particular, $\mathcal{G} \mathcal{R}_{E}=\operatorname{Gr}(E)$. We denote by $\mathcal{T} \mathcal{R}$ the class of acyclic graphs.

Theorem 16.3. The set $\mathcal{T} \mathcal{R}_{E}=\{G \in G r(E): G$ is acyclic $\}$ is closed in $\operatorname{Gr}(E)$.

A treeing $G$ of $E$ is an acyclic graphing of $E$.

Corollary 16.4. The set $\{G \in G r(E): G$ is a treeing of $E\}$ is a $G_{\delta}$ set in $G r(E)$. 
Similarly we define what it means to say that $G \in G r(E)$ is a graphing of $F \in S(E)$ (namely $G^{*}=F$ ) or a treeing of $F$. We thus have:

Corollary 16.5. The set $\{(G, F): G$ is a graphing of $F\}$ is $G_{\delta}$ in $G r(E) \times S(E)$. Similarly for $\{(G, F): G$ is a treeing of $F\}$. In particular $\{F \in S(E): F$ is treeable $\}$ is analytic in $S(E)$.

The following is a basic open problem.

Problem 16.6. Is $\{F \in S(E): F$ is treeable $\}$ Borel? Is there a Borel function $f:\{F \in S(E): F$ is treeable $\} \rightarrow G r(E)$ such that $f(F)$ is a treeing of $F$, if $F$ is treeable.

We next have the following fact, where for each $d \geq 1$, we let $\mathcal{G} \mathcal{R}_{d}=\{G \in$ $\mathcal{G R}: G$ has degree $\leq d\}$.

Proposition 16.7. The set $\mathcal{G R}_{d, E}=\{G \in G r(E): G$ has degree $\leq d\}$ is closed in $\operatorname{Gr}(E)$, for any $d \geq 1$.

Now let $\mathcal{B D} \mathcal{G}=\{G \in \mathcal{G} \mathcal{R}: G$ has bounded degree $\}$.

Corollary 16.8. The set $\mathcal{B D}_{\mathcal{E}}=\{G \in G r(E): G$ has bounded degree $\}$ is dense $F_{\sigma}$ in $\operatorname{Gr}(E)$. Moreover, if $E$ is aperiodic, then its complement is dense in $\operatorname{Gr}(E)$, so $\mathcal{B D}_{\mathcal{G}_{E}}$ is in $F_{\sigma} \backslash G_{\delta}$.

We also have, letting $\mathcal{I D} \mathcal{G}=\{G \in \mathcal{G} \mathcal{R}: G$ has infinite degree $\}$ :

Proposition 16.9. The set $\mathcal{I D}_{E}=\{G \in G r(E): G$ has infinite degree $\}$ is $G_{\delta}$ in $\operatorname{Gr}(E)$ and, if $E$ is aperiodic, it is dense in $\operatorname{Gr}(E)$.

Finally we have, letting $\mathcal{L F G}=\{G \in \mathcal{G} \mathcal{R}: G$ is locally finite $\}$, where a graph is locally finite if the degree of every vertex is finite.

Proposition 16.10. The set $\mathcal{L} \mathcal{F} \mathcal{G}_{E}=\{G \in G r(E): G$ is locally finite $\}$ is $F_{\sigma \delta}$ in $\operatorname{Gr}(E)$. Both $\mathcal{L} \mathcal{F} \mathcal{G}_{E}$ and its complement are dense in $\operatorname{Gr}(E)$, if $E$ is aperiodic. Moreover if $E$ is ergodic, $\mathcal{L} \mathcal{F} \mathcal{G}_{E}$ is in $F_{\sigma \delta} \backslash G_{\delta \sigma}$.

Denote by $C_{\mu}(G)=C(G)$ the cost of $G$, i.e., $C(G)=\frac{1}{2} \int \operatorname{deg}_{G}(x) d \mu(x) \in[0, \infty]$.

Proposition 16.11. The function $\operatorname{Gr}(E) \ni G \mapsto C(G)$ is lower semicontinuous. In particular, $\{G \in G r(E): C(G)=\infty\}$ is $G_{\delta}$.

Theorem 16.12. If $E$ is aperiodic, the set $\{G \in G r(E): C(G)=\infty\}$ is dense and therefore the generic $G \in G r(E)$ is a graphing of $E$ of infinite cost.

It is also clear that the $F_{\sigma}$ set $\{G \in G r(E): C(G)<\infty\}$ is dense, since every $G$ can be written as the union of an increasing sequence $G_{n}$ with $C\left(G_{n}\right)=\frac{1}{2} M\left(G_{n}\right)<$ $\infty$. In particular, it follows that $\{G \in G r(E): C(G)<\infty\}$ is in $F_{\sigma} \backslash G_{\delta}$.

Finally we have the following result concerning locally finite graphings of equivalence relations (see [JKL, Theorem 3.12]).

Proposition 16.13. There is a Borel function $\Lambda: S(E) \rightarrow G r(E)$ such that for any $F \in S(E), \Lambda(F)$ is a locally finite graphing of $F$. 


\section{Treeability}

Recall that, by Theorem 16.3, the set

$$
\mathcal{T} \mathcal{R}_{E}=\{G \in G r(E): G \text { is acyclic }\} .
$$

is closed in $\operatorname{Gr}(E)$. By Corollary 16.4, the set

$$
\operatorname{Treeing}(E)=\left\{G \in \mathcal{T}_{\mathcal{R}_{E}}: G \text { is a treeing of } E\right\}
$$

is $G_{\delta}$ in $\mathcal{T R}_{E}$. If $E$ is not treeable, clearly this set is empty.

Problem 17.1. If $E$ is ergodic, treeable, is Treeing $(E)$ dense in $\mathcal{T R}_{E}$ ?

We first note the following:

Proposition 17.2. If there is $G \in \mathcal{T R}_{E}$ with $C(G)=\infty$, then $\left\{G \in \mathcal{T} \mathcal{R}_{E}: C(G)=\right.$ $\infty$ is dense in $\mathcal{T} \mathcal{R}_{E}$.

Recall that $C(E)$ denotes the cost of the equivalence relation $E$.

Proposition 17.3. Let $E$ be ergodic with $C(E)>1$. Then the set $\left\{G \in \mathcal{T}_{\mathcal{R}_{E}}: C(G)=\right.$ $\infty\}$ is dense $G_{\delta}$ in $\mathcal{T R}_{E}$. In particular if $1<C(E)<\infty$, then the generic $G \in \mathcal{T} \mathcal{R}_{E}$ is not a treeing of $E$.

Thus Problem 17.1 has a negative answer if $C(E)<\infty$, but $E$ is not hyperfinite (in which case $C(E)>1$ ). We next show that it has a positive answer if $E$ is hyperfinite.

Proposition 17.4. Let $E$ be ergodic, hyperfinite. Then Treeing $(E)$ is dense in $\mathcal{T R}_{E}$ and thus the generic $G \in \mathcal{T} \mathcal{R}_{E}$ is a treeing of $E$.

Corollary 17.5. Let $E$ be ergodic, with finite cost. Then $E$ is hyperfinite iff the generic $G \in \mathcal{T} \mathcal{R}_{E}$ is a treeing of $E$.

Thus the only remaining open case of Problem 17.1 is when $C(E)=\infty$.

There is actually a strengthening of Proposition 17.4, proved by A. Tserunyan. We will use below the following notation and terminology,.

We call $G \in G r(E)$ finite, smooth, hyperfinite if $G^{*}$ is, resp., finite, smooth, hyperfinite. Let $\mathcal{F} \mathcal{T} \mathcal{R}_{E}, \mathcal{S} \mathcal{T} \mathcal{R}_{E}$ and $\mathcal{H} \mathcal{T} \mathcal{R}_{E}$ denote, resp., the set of finite, smooth, hyperfinite $G \in \mathcal{T} \mathcal{R}_{E}$. Note that by Theorem $15.3, \mathcal{H} \mathcal{T} \mathcal{R}_{E}$ is closed and $\overline{\mathcal{F} \mathcal{T} \mathcal{R}_{E}}=$ $\overline{\mathcal{S T} \mathcal{R}_{E}}=\mathcal{H} \mathcal{T} \mathcal{R}_{E}$.

Proposition 17.6 (Tserunyan). Let $E$ be aperiodic and treeable. For any $G_{0} \in$ $\mathcal{S T} \mathcal{R}_{E}$ and $T_{1}, \ldots, T_{m} \in[E]$, there is a treeing $G \supseteq G_{0}$ of $E$ such that $A_{T_{i}, G}=$ $A_{T_{i}, G_{0}}$, for all $i$.

Theorem 17.7 (Tserunyan). Let $E$ be aperiodic and treeable. Then we have $\mathcal{H} \mathcal{R}_{E} \subseteq \overline{\text { Treeing }(E)}$. In particular, if $E$ is hyperfinite, then Treeing $(E)$ is dense in $\mathcal{T R}_{E}$.

Remark 17.8. Proposition 17.6 cannot be extended to graphs $G_{0} \in \mathcal{H} \mathcal{T} \mathcal{R}_{E}$, even if we drop the requirement about the $T_{i}$ 's.

Let $\operatorname{SubTreeing}(E)$ denote the set of all graphs in $\mathcal{T R}_{E}$ that are contained in treeings of $E$, i.e.,

$$
\operatorname{SubTreeing}(E)=\left\{G_{0} \in \mathcal{T R}_{E}: \exists G \in \operatorname{Treeing}(E)\left(G \supseteq G_{0}\right)\right\} .
$$


Proposition 17.9 (Tserunyan). Let E be treeable. Then

$$
\mathcal{S T} \mathcal{R}_{E} \subseteq \text { SubTreeing }(E) .
$$

Therefore, in particular, we have $\mathcal{H} \mathcal{T} \mathcal{R}_{E} \subseteq \overline{\text { SubTreeing }(E)}$.

Proposition 17.10. Let $G_{0} \in \operatorname{SubTreeing}(E)$.

(a) (Tserunyan) For any Borel set $A \subseteq X, G_{0} \mid A \in \operatorname{SubTreeing}(E \mid A)$.

(b) (Conley) For a Borel equivalence relation $F$ with $G_{0}^{*} \subseteq F \subseteq E, G_{0} \in$ $\operatorname{SubTreeing}(F)$.

Theorem 17.11 (Tserunyan). For any $G_{0} \in \operatorname{SubTreeing}(E)$ and automorphisms $T_{1}, \ldots, T_{m} \in[E]$, there is a treeing $G \supseteq G_{0}$ of $E$ such that $A_{T_{i}, G}=A_{T_{i}, G_{0}}$, for all $i$. In particular, Treeing $(E)$ is dense in SubTreeing $(E)$ and hence $\overline{\text { Treeing }(E)}=$ $\overline{\text { SubTreeing }(E)}$.

Let $\operatorname{Max} \operatorname{Tr}(E)$ denote the set of maximal (under inclusion) graphs in $\mathcal{T} \mathcal{R}_{E}$; that is,

$$
\operatorname{Max} \operatorname{Tr}(E)=\left\{G \in \mathcal{T} \mathcal{R}_{E}: \forall G^{\prime} \in \mathcal{T} \mathcal{R}_{E}\left(G^{\prime} \supseteq G \Rightarrow G^{\prime}=G\right\} .\right.
$$

Theorem 17.12 (Tserunyan). Let $E$ be a (not necessarily treeable) equivalence relation. Then for any $G_{0} \in \mathcal{T R}_{E}$ and $T_{1}, \ldots, T_{m} \in[E]$, there is $G \in \operatorname{Max} \operatorname{Tr}(E)$ such that $G \supseteq G_{0}$ and $A_{T_{i}, G}=A_{T_{i}, G_{0}}$, for all $i$. In particular, $\operatorname{Max} \operatorname{Tr}(E)$ is dense in $\mathcal{T} \mathcal{R}_{E}$.

Let $\operatorname{SubTreeing}(E)$ denote the set of all graphs in $\operatorname{SubTreeing}(E)$ that are not treeings anywhere; i.e.

$$
\text { SubTreeing }^{*}(E)=\left\{G \in \operatorname{SubTreeing}(E): \mu\left(\left\{x \in X:[x]_{E}=[x]_{G^{*}}\right\}\right)=0\right\} .
$$

Proposition 17.13 (Tserunyan). For any $G \in \operatorname{Treeing}(E)$ and $\epsilon>0$, there is $G_{0} \in S_{\text {SubTreeing* }}(E)$ with $G_{0} \subseteq G$ such that $\mu\left(A_{T, G} \backslash A_{T, G_{0}}\right)<\epsilon$, for all $T \in[E]$. In particular, SubTreeing* $(E)$ is dense in Treeing $(E)$.

Theorem 17.11 and Proposition 17.13 together imply:

Theorem $\mathbf{1 7 . 1 4}$ (Tserunyan). $\overline{\text { SubTreeing* }(E)}=\overline{\text { Treeing }(E)}=$ $\overline{\operatorname{SubTreeing}(E)}$.

\section{References}

[AE] M. Abért and G. Elek, The space of actions, partition metric and combinatorial rigidity, arXiv:1108.21471v1.

[AP] C. Anantharaman and S. Popa, An introduction to $\mathrm{II}_{1}$ factors, preprint, 2017.

[Bo] L. Bowen, Every countably infinite group is almost Ornstein, Contemp. Math., 567 (2012), 67-78.

[Bo1] L. Bowen, Simple and large equivalence relations, Proc. Amer. Math. Soc., 145(1) (2017), 215-224.

[BJ] C.M. Boykin and S. Jackson, Borel boundedness and the lattice rounding property, Contemp. Math., 425 (2007), 113-126.

[CK] R. Chen and A.S. Kechris, Structurable equivalence relations, Fund. Math., 242 (2018), 109-185. 
[CKT] C.T. Conley, A.S. Kechris and R.D. Tucker-Drob, Ultraproducts of measure preserving actions and graph combinatorics, Ergod. Th. \& Dynam. Sys., 33, (2013), 334-374.

[D] J. Dugundji, Topology, Allyn and Bacon, 1966.

[EL] G.Elek and G. Lippner, Sofic equivalence relations, J. Funct. Anal., 258 (2010), 1692-1708.

[F] R.H. Farrell, Representation of invariant measures, Illinois. J. Math., 6 (1962), 447-467.

[FSZ] J. Feldman, C.E. Sutherland and R.J. Zimmer, Subrelations of ergodic equivalence relations, Ergod. Th. and Dynam. Sys., 9 (1989), 239-269.

[GT] D. Gaboriau and R.D. Tucker-Drob, Approximations of standard equivalence relations and Bernoulli percolation at $p_{u}$, C.R. Acad. Sci. Paris Sér. I Math, 354(11) (2016), 1114-1118.

[Gl] E. Glasner, Ergodic Theory via Joinings, Amer. Math. Soc., 2003.

[GW] E. Glasner and B. Weiss, Kazhdan's property T and the geometry of the collection of invariant measures, Geom. Funct. Anal., 7 (1997), 917-935.

[HK] G. Hjorth and A.S. Kechris, Rigidity theorems for actions of product groups and countable Borel equivalence relations, Memoirs Amer. Math. Soc., 177 (833) (2005).

[I] A. Ioana, Rigidity for von Neumann algebras, Proc. of ICM, Rio de Janeiro, 2018, Vol III, Invited Lectures, World Scientific (2018), 1639-1672.

[IKT] A. Ioana, A.S. Kechris, and T. Tsankov, Subequivalence relations and positive-definite functions, Groups Geom. Dyn., 3 (2009), 579-625.

[JKL] S. Jackson, A.S. Kechris, and A. Louveau, Countable Borel equivalence relations, J. Math. Logic, 2 (1) (2002), 1-80.

[JS] V.F.R. Jones and K. Schmidt, Asymptotically invariant sequences and approximate finiteness, Amer. J. Math., 109 (1987), 91-114.

[K] A.S. Kechris, Global aspects of ergodic group actions, Amer. Math. Soc., 2010.

[K1] A.S. Kechris, Weak containment in the space of actions of a free group, Israel J. Math., 189 (2012), 461-507.

[K2] A.S. Kechris, Classical Descriptive Set Theory, Springer, 1995.

[K3] A.S. Kechris, The spaces of measure preserving equivalences and graphs, preprint, 2021. http://www.math.caltech.edu/ kechris/

[KM] A.S. Kechris and B.D. Miller, Topics in Orbit Equivalence, Springer, 2004.

[KT] J. Kittrell and T. Tsankov, Topological properties of full groups, Ergod. Th. and Dynam. Sys., 30 (2010), 525-545.

[LeM] F. Le Maître, The number of topological generators for full groups of ergodic equivalence relations, Invent. Math., 198(2) (2014), 261-268.

[LeM1] F. Le Maître, Dense orbit on the space of subequivalence relations of the hyperfinite ergodic equivalence relation, preprint, 2018.

[L] L. Lovász, Large Networks and Graph Limits, Amer. Math. Soc., 2012.

[Po] S. Popa, Cocycle and orbit equivalence superrigidity for malleable actions of w-rigid groups, Invent. Math., 170(2) (2007), 243-295.

[T] A. D. Törnquist, The Borel complexity of orbit equivalence, Ph.D. Thesis, UCLA, 2005.

[T-D] R.D. Tucker-Drob, Shift-minimal groups, fixed price 1, and the unique trace property, arXiv:1211.6395v3. 
[Va] S. Vaes, Explicit computations of all finite index bimodules for a family of $I I_{1}$ factors, Ann. Scient. Ecole Normal Sup., 4(1) (2008), 743-788.

[Z] R.J. Zimmer, Ergodic theory and semisimple groups, Birkhäuser, 1984.

Department of Mathematics

California Institute of Technology

Pasadena, CA 91125

kechris@caltech.edu 